\title{
Review Article \\ NSAIDs and Cardiovascular Diseases: Role of Reactive Oxygen Species
}

\author{
Rajeshwary Ghosh, ${ }^{1}$ Azra Alajbegovic, ${ }^{1}$ and Aldrin V. Gomes ${ }^{1,2}$ \\ ${ }^{1}$ Department of Neurobiology, Physiology, and Behavior, University of California, Davis, CA 95616, USA \\ ${ }^{2}$ Department of Physiology and Membrane Biology, University of California, Davis, CA 95616, USA \\ Correspondence should be addressed to Aldrin V. Gomes; avgomes@ucdavis.edu
}

Received 22 December 2014; Revised 3 March 2015; Accepted 3 March 2015

Academic Editor: Mark J. Crabtree

Copyright (C) 2015 Rajeshwary Ghosh et al. This is an open access article distributed under the Creative Commons Attribution License, which permits unrestricted use, distribution, and reproduction in any medium, provided the original work is properly cited.

\begin{abstract}
Nonsteroidal anti-inflammatory drugs (NSAIDs) are the most commonly used drugs worldwide. NSAIDs are used for a variety of conditions including pain, rheumatoid arthritis, and musculoskeletal disorders. The beneficial effects of NSAIDs in reducing or relieving pain are well established, and other benefits such as reducing inflammation and anticancer effects are also documented. The undesirable side effects of NSAIDs include ulcers, internal bleeding, kidney failure, and increased risk of heart attack and stroke. Some of these side effects may be due to the oxidative stress induced by NSAIDs in different tissues. NSAIDs have been shown to induce reactive oxygen species (ROS) in different cell types including cardiac and cardiovascular related cells. Increases in ROS result in increased levels of oxidized proteins which alters key intracellular signaling pathways. One of these key pathways is apoptosis which causes cell death when significantly activated. This review discusses the relationship between NSAIDs and cardiovascular diseases (CVD) and the role of NSAID-induced ROS in CVD.
\end{abstract}

\section{Introduction}

Nonsteroidal anti-inflammatory drugs (NSAIDs) are the most widely used over-the-counter drugs as well as the most prescribed class of drugs for a variety of conditions including pains, rheumatoid arthritis, osteoarthritis, musculoskeletal disorders, and other comorbid conditions [1]. Millions of people suffer from pain resulting in the prolonged use of NSAIDs being common. Besides reducing or relieving pain NSAIDs have been shown to be useful as anticancer agents in various kinds of cancers [2-4]. However, NSAIDs also have undesirable side effects including ulcers [5], bleeding [6], kidney failure $[7,8]$, and increased risk of heart attack and stroke $[8,9]$. One of the mechanisms which has been associated with the adverse effects of NSAIDs is the generation of oxidative stress. The present review focuses on NSAIDs-induced ROS generation leading to cardiovascular diseases (CVD).

\section{Types of NSAIDs}

NSAIDs may be classified according to their mechanism of action. Nonselective NSAIDs like ibuprofen and naproxen, which comprise one class, inhibit both cyclooxygenase-1 (COX-1) and cyclooxygenase-2 (COX-2) enzymes. A second class of NSAIDs (celecoxib and rofecoxib) targets only the COX-2 pathway and is termed as COX-2 selective inhibitors (also known as coxibs). COX selectivity is one of the determining factors that is considered when administrating NSAIDs to a patient. Administration of nonselective NSAIDs has been associated with side effects like peptic ulcer disease and gastrointestinal bleeding [10]. COX-2 selective NSAIDs have been shown to exhibit gastroprotective effects unlike the nonselective NSAIDs and are thus useful in patients with painful gastrointestinal conditions [10-12]. Another class of semiselective NSAIDs (indomethacin, meloxicam, and diclofenac) have a higher affinity for COX-2 but tend to inhibit the COX-1 pathway also [13]. However, irrespective of their mechanism of action, prolonged exposure to any class of NSAIDs has been shown to have potential adverse effects on cardiovascular events in patients with or without preexisting cardiovascular conditions, depending on the duration and dosage of these drugs [14, 15] (Table 1). Patients with preexisting cardiovascular conditions such as coronary 


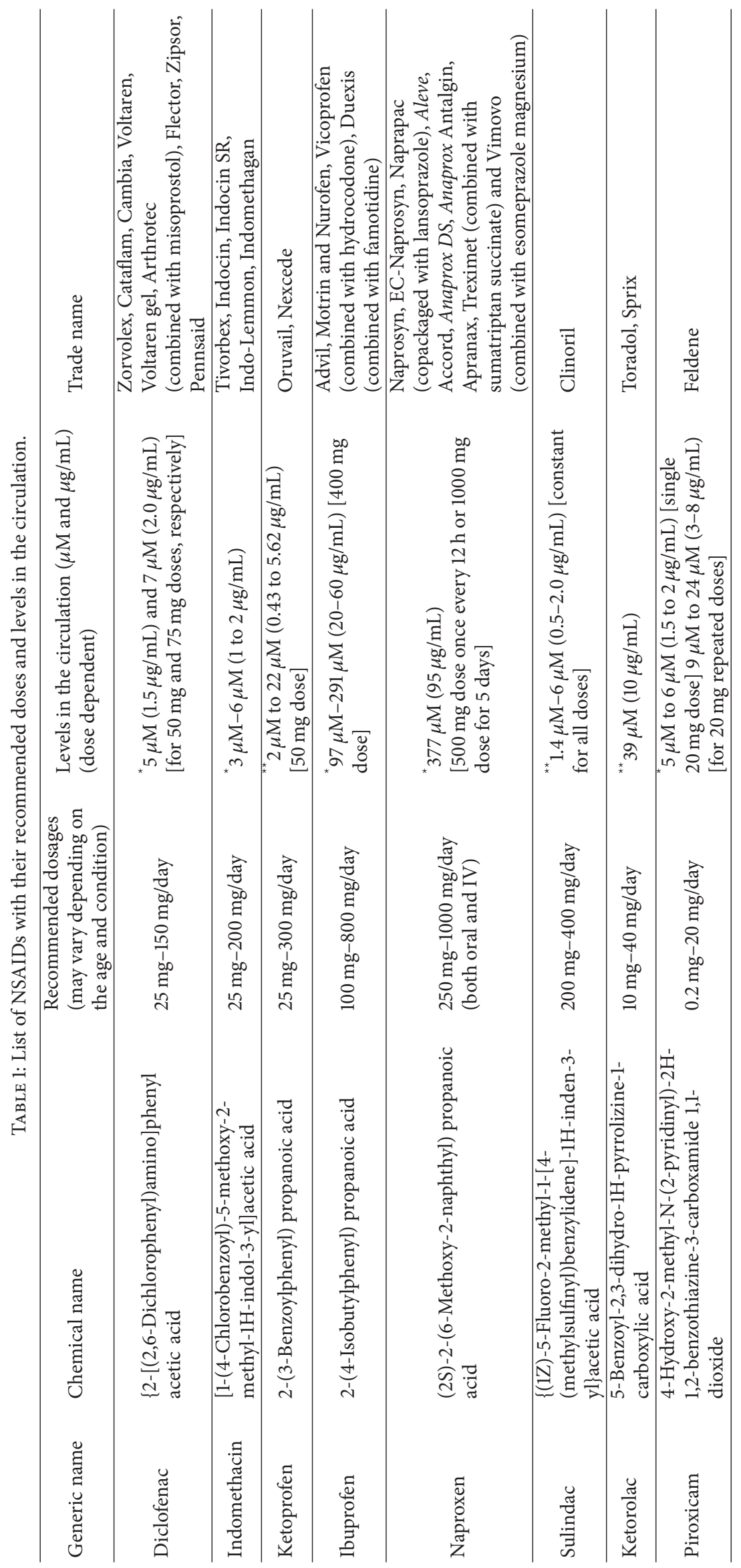




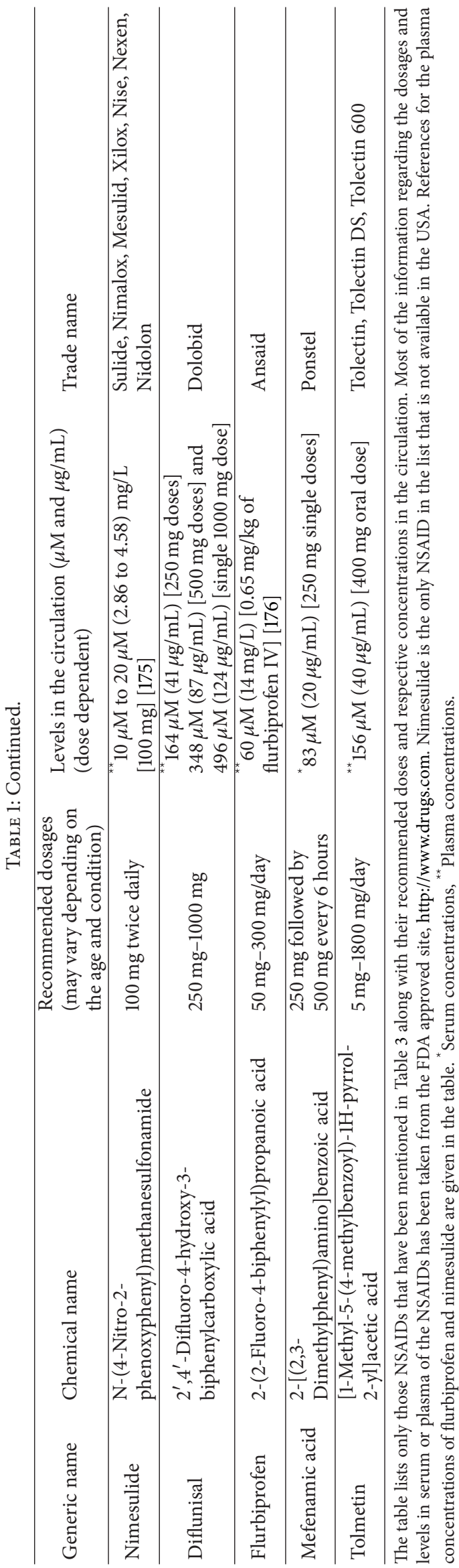




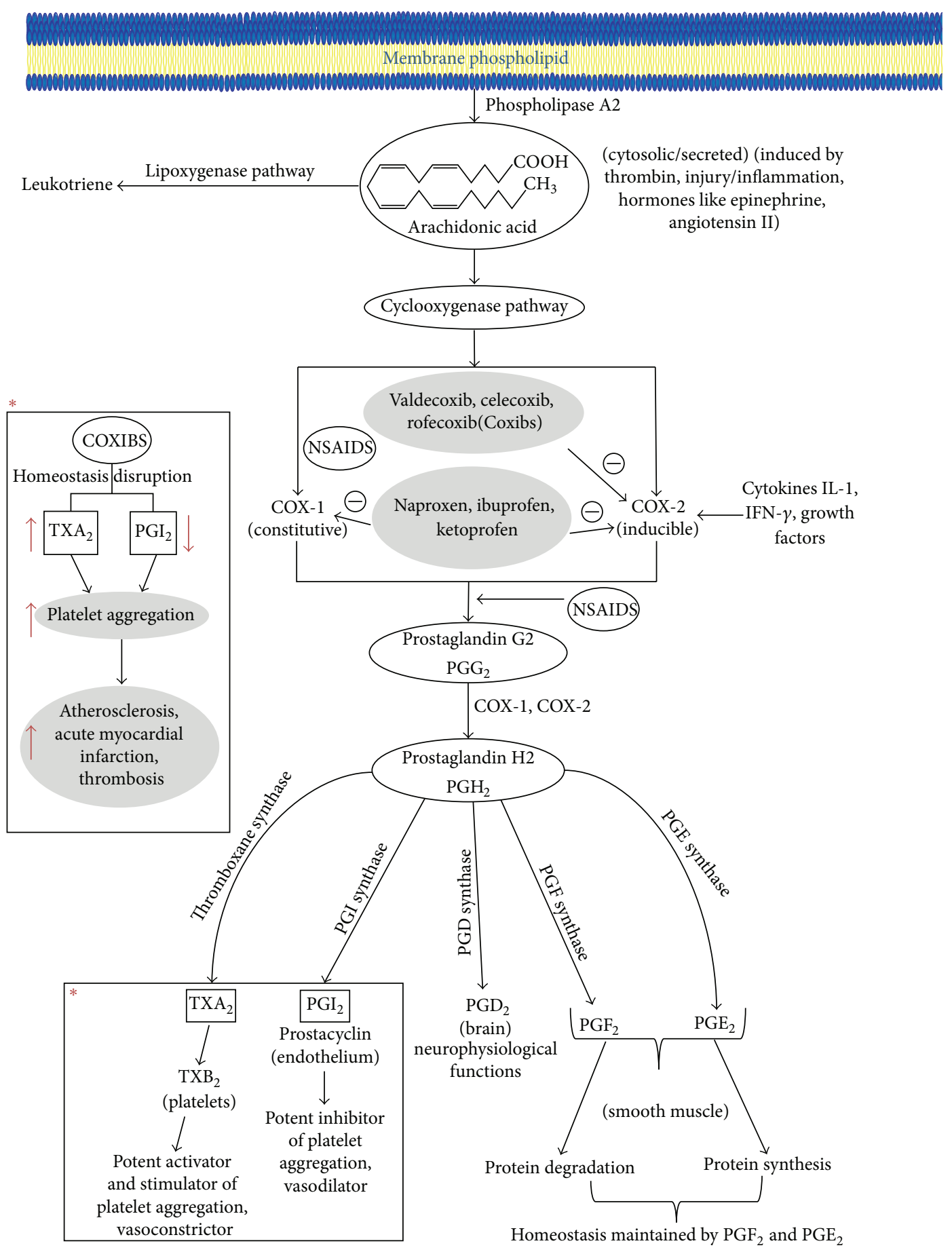

FIGURE 1: Inhibition of cyclooxygenase pathway by NSAIDs. Coxibs as well as nonselective NSAIDs inhibit the formation of the metabolites of the cyclooxygenase pathway thereby disrupting the homeostasis maintained by these metabolites. Coxibs cause an imbalance between the levels of thromboxane and prostacyclin being more favorable towards thromboxane and decreasing prostacyclin levels leading to the aggregation of platelets and causing thrombosis.

artery disease, hypertension, and history of stroke are at the greatest risk of cardiovascular events after taking NSAIDs $[14,15]$. Patients who have recently had cardiovascular bypass surgery are advised not to take NSAIDs due to a high risk of heart attacks $[16,17]$. The increased selectivity for COX2 has also been reported to increase the risk of various CVD $[18,19]$. Meta-analyses of several trials have shown that coxibs are associated with a high risk of atherothrombotic vascular events [20].

\section{Mechanism of Action of NSAIDs}

NSAIDs exert their pain relieving effect mainly by inhibiting the cyclooxygenase pathway (Figure 1). This pathway 
is responsible for the conversion of arachidonic acid to prostaglandins and thromboxanes [21]. Although COX is officially known as prostaglandin-endoperoxide synthase (PTGS) the abbreviation "COX" is commonly used for cyclooxygenase- 1 and cyclooxygenase- 2 in medicine. In genetics, the abbreviation PTGS is officially used for the cyclooxygenase family of genes and proteins to prevent ambiguity with the cytochrome c oxidase family of genes and proteins which are also abbreviated COX. Arachidonic acid is the main precursor to the formation of various eicosanoids in the cyclooxygenase pathway. Of all the metabolites formed in the arachidonic acid metabolism, thromboxane A2 in the platelets is the major product which along with prostacyclin (prostaglandin I2) maintains vascular homeostasis [22] (Figure 1). Both these eicosanoids (thromboxane A2 and prostaglandin I2) have opposing effects. While thromboxane is well known for its role in vasoconstriction and aggregation of platelets, prostacyclin is important for platelet aggregation inhibition and vasodilation.

The COX enzyme is present as two isoforms, each with distinct functions: COX-1 is constitutively expressed in the stomach, kidneys, intestinal mucosa, and other tissues [23]. It protects the mucosal lining of the stomach and plays an important role in vasoconstriction and platelet aggregation [23]. On the other hand the inducible COX-2 is upregulated during times of inflammation where it causes vasodilation [24]. COX-1 and COX-2 are similar in molecular weights, 70 and $72 \mathrm{kDa}$, respectively, and show $65 \%$ homology with near-identical catalytic sites (based upon information from UniProtKB/Swiss-Prot database). The critical difference between the isoenzymes, which permits the selective inhibition of each isoform, is the substitution of isoleucine 523 in COX-1 with valine in COX-2 [25]. The presence of valine, which is a smaller amino acid than isoleucine, allows drugs entrance to a hydrophobic side-pocket only accessible in COX-2. Expression of both isoforms, COX-1 and COX-2, may be upregulated and downregulated under various pathological conditions [26]. It is likely that the classification of the COX enzymes into two isoforms was an oversimplification [26] as COX-2 may be constitutively expressed in the brain [27], kidney [28], and testes [29]. In fact immunohistochemical studies have revealed the constitutive expression of COX-2 mRNA in the lung, thyroid gland, spleen, and adipose tissue, which was greater than COX-1 in these tissues, and in the liver both isoforms were expressed equally [29]. Therefore the idea that COX-2 can only be expressed under inducible conditions is unlikely since recent evidence suggests their occurrence in various human tissues under normal conditions.

Coxibs disrupt the balance between the levels of thromboxane A2 and prostaglandin I2 leading to atherosclerosis, thrombosis, and other cardiovascular complications. Coxibs, through their selective inhibition of COX-2, inhibit endothelial cell synthesis of prostacyclin [30]. In the ApoE-/mice (model for atherosclerosis), deletion of the prostacyclin receptor increased atherogenesis with no such effect observed in thromboxane receptor deleted mice [30]. Apart from its role in the inhibition of cyclooxygenase pathway, NSAIDs have been shown to cause cell death by the inhibition of the Akt signaling pathway [31], downregulation of the NF- $\kappa \mathrm{B}$ pathway [32], downregulation of the Bcl pathway [33], upregulation of the nonsteroidal activated gene-1 [34], and altering the p53 pathway [35], all of which have been suggested to be involved in apoptosis [36]. Apoptosis (programmed cell death) induced by NSAIDs has been suggested to be due to oxidative stress caused by increased generation of reactive oxygen species (ROS) [37].

A series of mechanisms are involved wherein NSAIDs exert their cardiotoxic effects and cause various cardiac conditions. Various non-NSAID drugs like doxorubicin, azidothymidine, and cisplatin have been shown to induce oxidative stress as a consequence of elevated ROS levels [38]. Doxorubicin, for example, induced cardiotoxicity through DNA damage and apoptosis in cardiac cells as a result of oxidative stress which were reduced by the antioxidant effect of statin [37]. It is possible that the oxidative stress induced by NSAIDs, which is known to cause apoptosis and cell death, is significantly involved in causing cardiovascular dysfunction (Figure 2).

\section{Incidences of CVD Induced by NSAIDs}

Various clinical trials have been made during the past few years regarding the safety and effectiveness of NSAIDs in CVD (Table 2). Most trials focused on the CVD outcome of NSAID use in patients with a previous history of cardiovascular disease. Few trials were carried out on patients with no history of CVD. An important finding is that not only nonselective NSAIDs lead to the development of hypertension in both normotensive and hypertensive individuals [39], but their use interferes with the antihypertensive medications except for the calcium channel blockers [40]. The risk of atrial fibrillation, heart failure, myocardial infarction, and other cardiovascular conditions also increased in patients with a history of these pathological conditions (Table 2).

The CVD related outcomes in patients enrolled in the REACH (REduction of Atherothrombosis for Continued Health) registry showed that in patients with established stable atherothrombosis, use of NSAIDs increased the incidences of myocardial infarction and cerebrovascular conditions [41]. Additionally, this paper reported that the use of NSAIDs with other antiplatelet drugs (except aspirin) increased the rate of cardiovascular events like cardiovascular death, myocardial infarction, and stroke [41]. Although a few studies suggest that COX selectivity does not seem to be a determining factor for myocardial infarction [13, 42], several studies suggest that coxibs elevate the rate of incidences of CVD compared to nonselective NSAIDs [18-20]. Several clinical trials have been completed and are still ongoing, but some inconsistencies in the results exist regarding the effect of different types of NSAIDs on cardiovascular outcomes (Table 2 ). One meta-analysis report found that $\sim 75 \%$ studies investigating the cardiovascular risk in new NSAID users reported an increase in the occurrence of cardiovascular diseases within the first month of NSAID use [43]. Overall, the different trials showed that several NSAIDs increased the risk of CVD both at low and high doses [41, 43-45]. Targeted inhibition of COX-2, even for a short term, was 


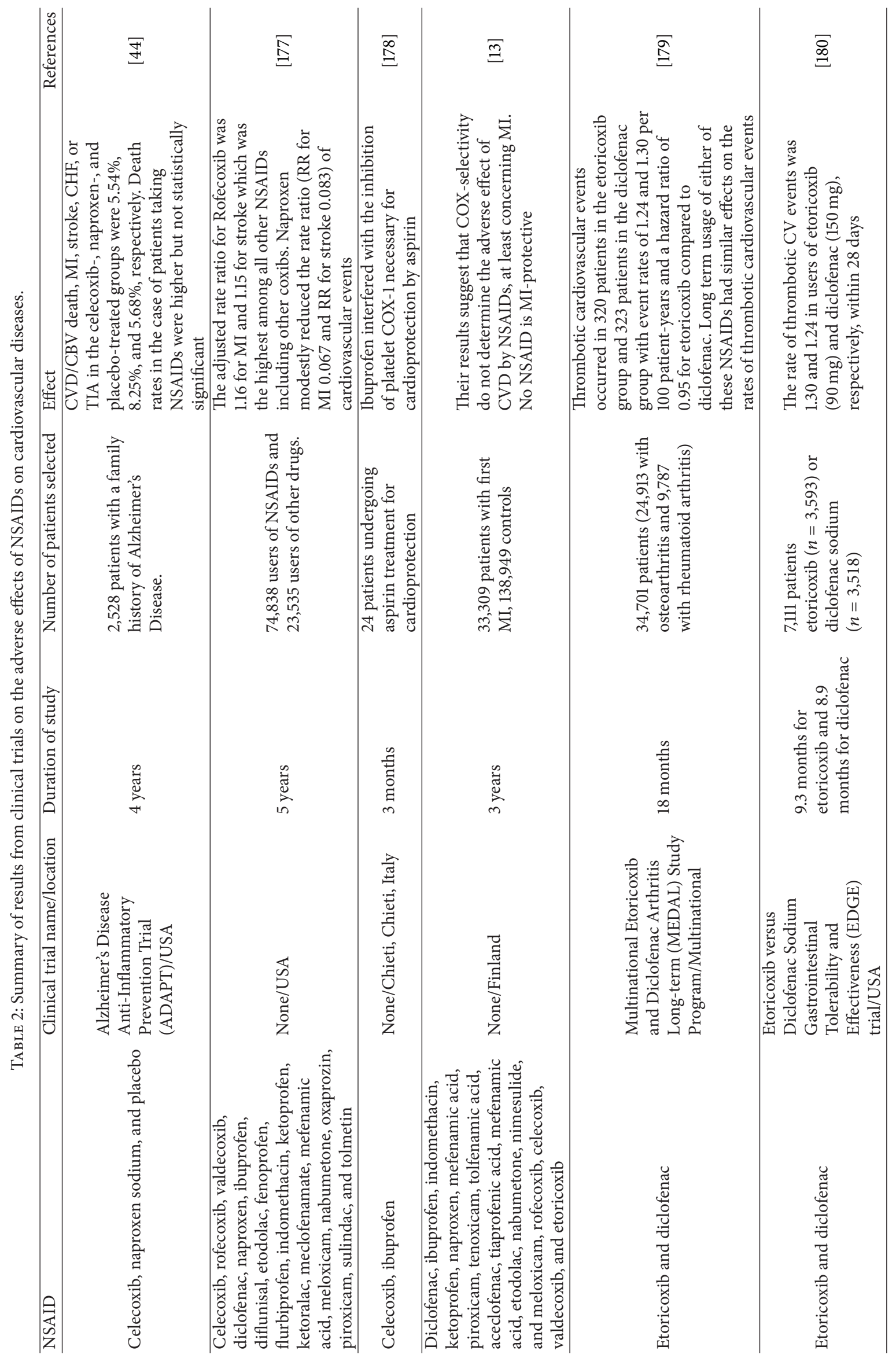




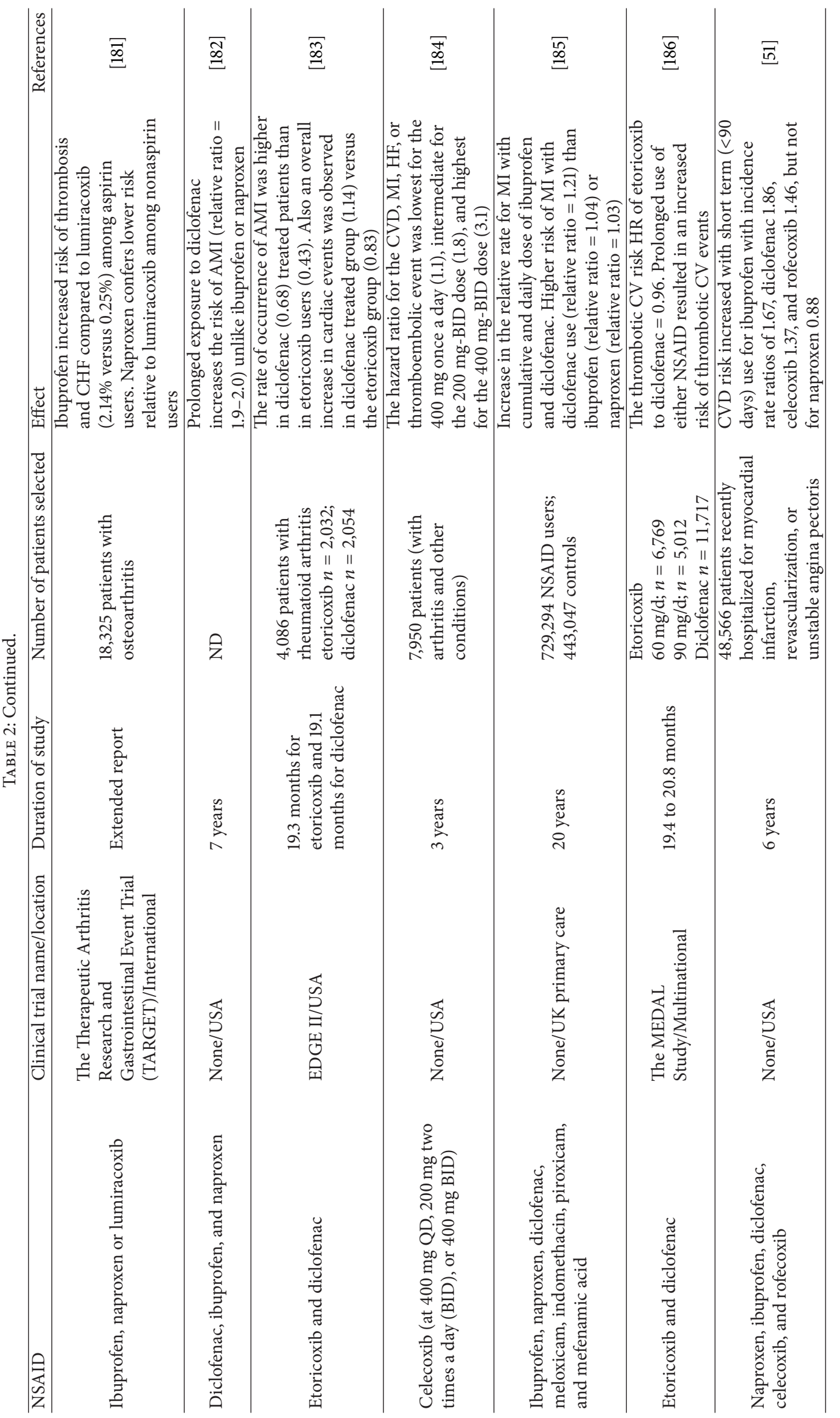




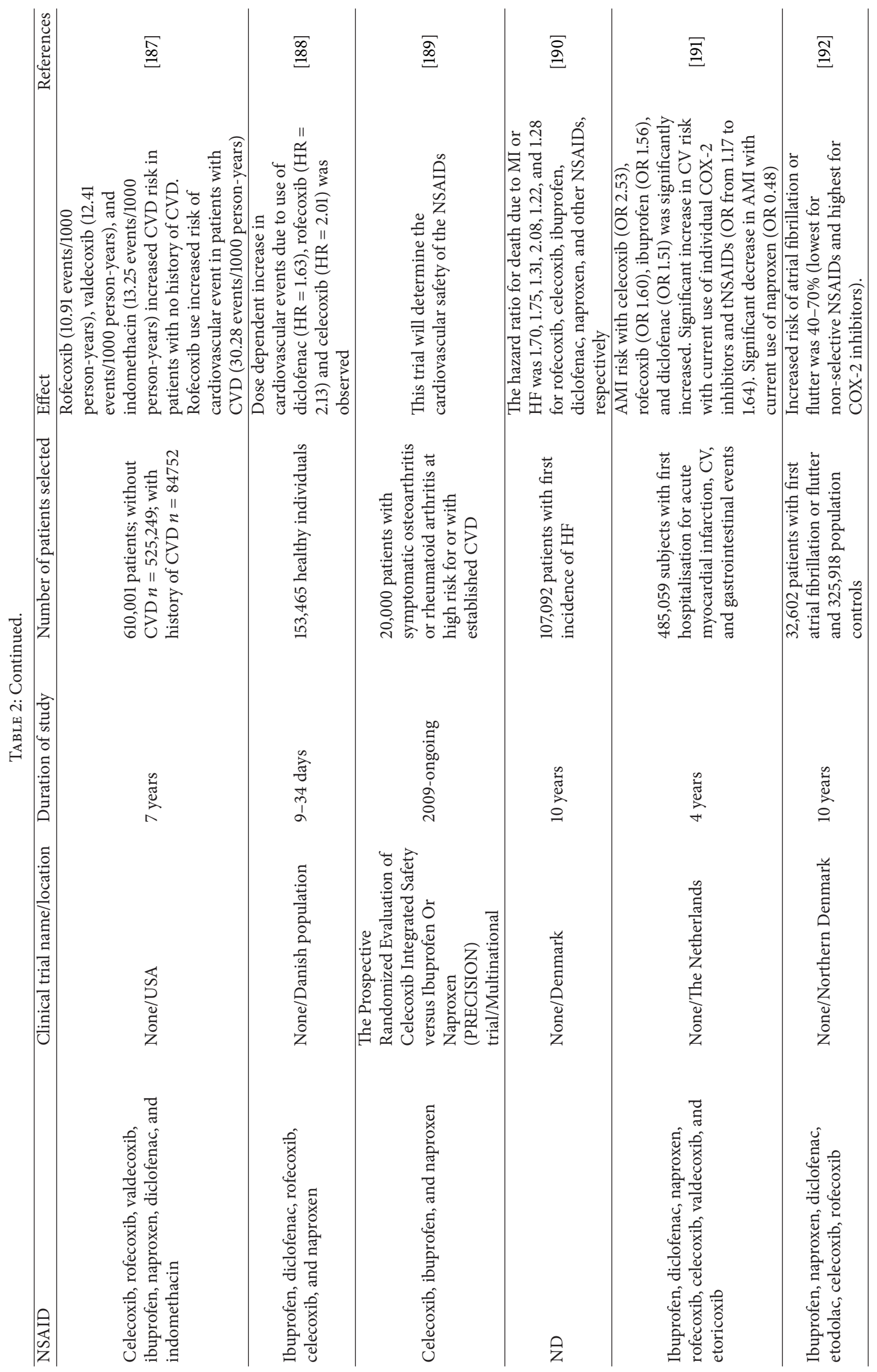




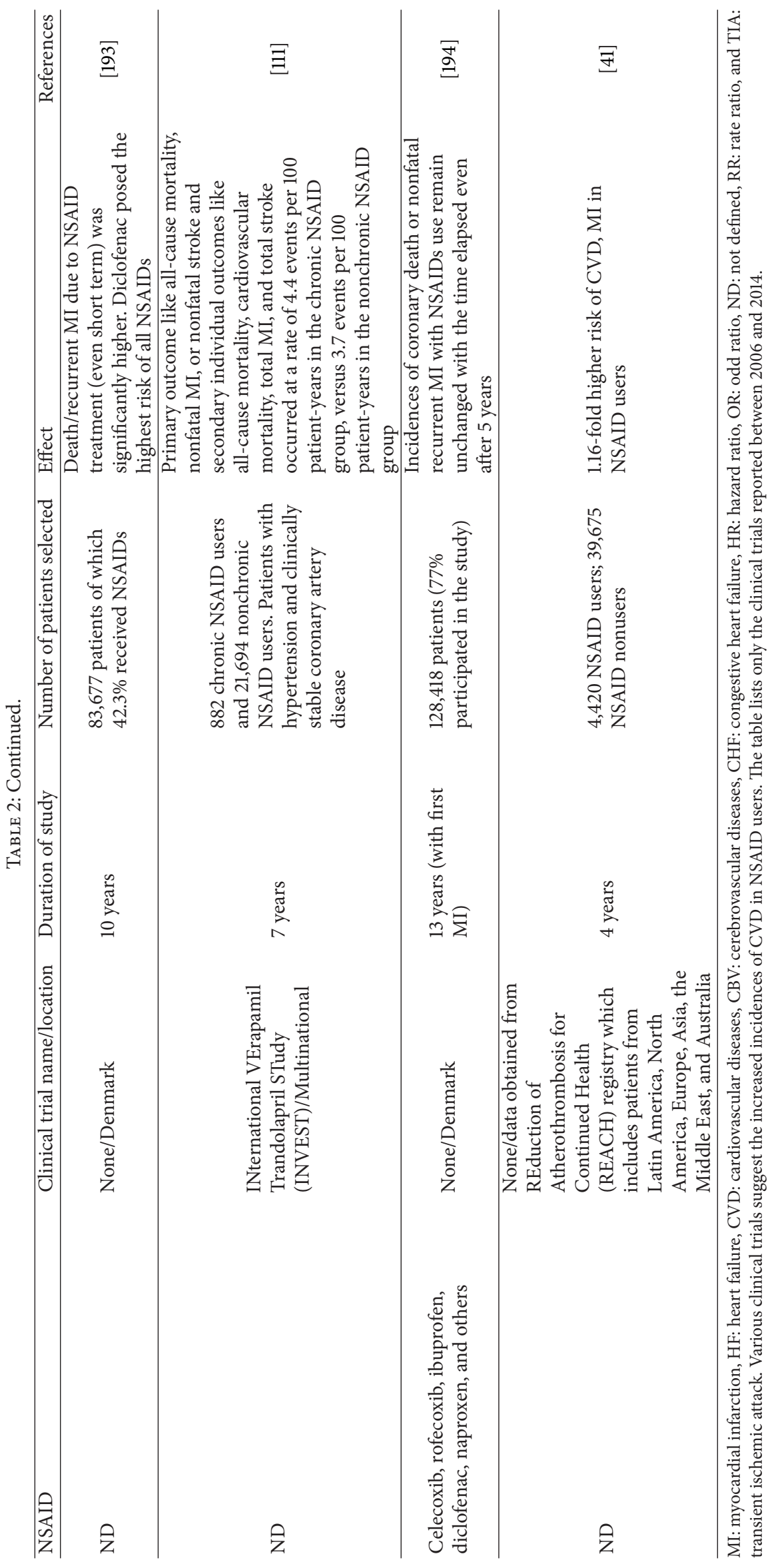




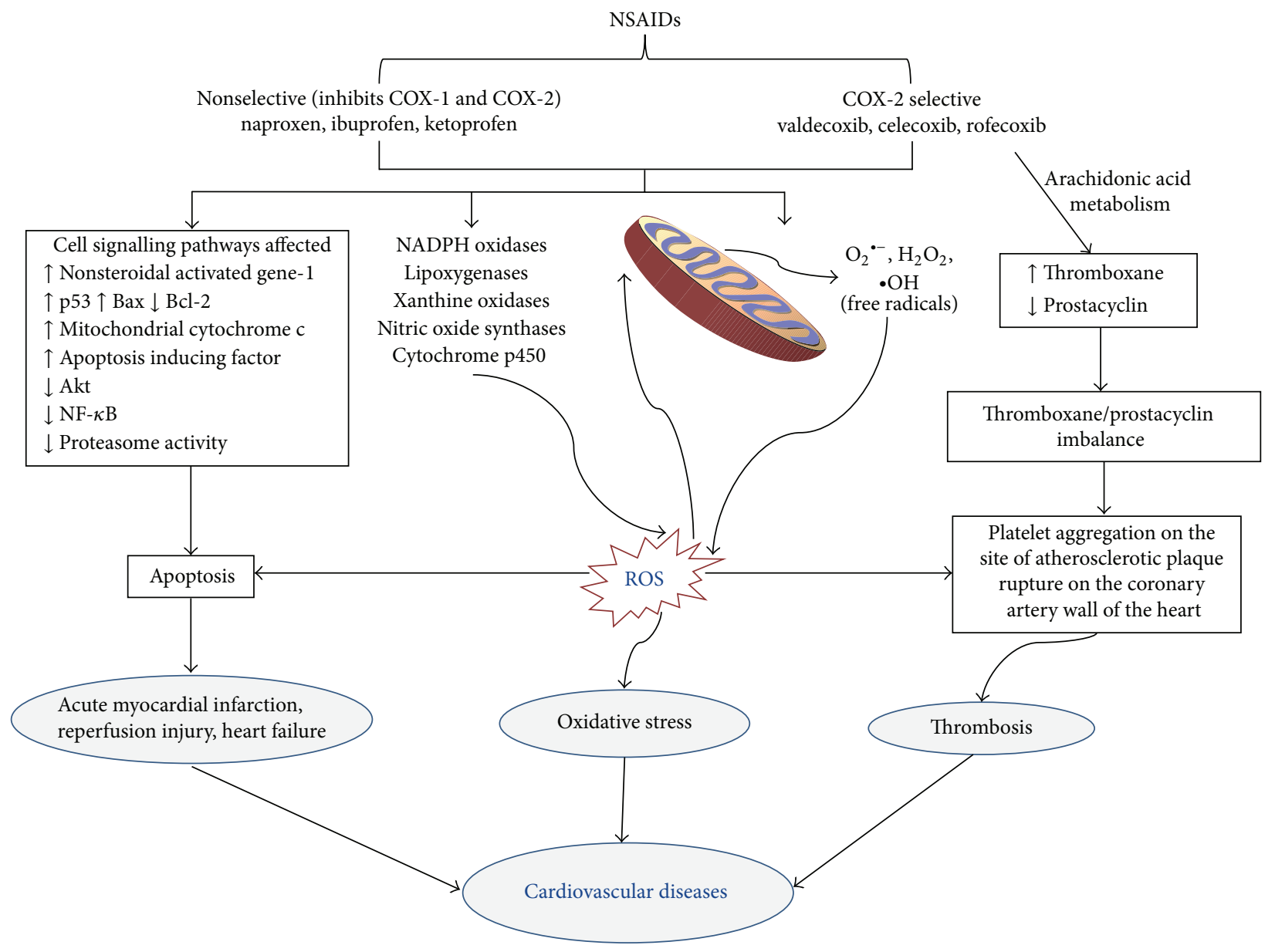

FIGURE 2: Pathways involved in the development of cardiovascular diseases by NSAIDS. The figure shows the upregulation and downregulation of various pathways by NSAIDs leading to the development of CVD. Mitochondria play a major role in the generation of ROS induced by NSAIDs followed by oxidative stress and finally CVD.

found to increase the risk of atherothrombosis [46]. The COX-2 selective NSAID rofecoxib at low doses (50 mg daily) increased the occurrences of myocardial infarction by $0.5 \%$ in approximately 8000 patients with rheumatoid arthritis compared to $0.1 \%$ in those treated with naproxen $(500 \mathrm{mg}$ twice daily), indicating a severe thrombogenic effect of rofecoxib compared to naproxen [47]. The main increased risk of cardiovascular events associated with COX-2 inhibitors was an increased risk of myocardial infarction [46].

The report of Adenomatous Polyp Prevention on Vioxx (APPROVe) trial on the adverse effect of rofecoxib on CVD ultimately led to the discontinuation of this drug in several countries [48]. Increased thromboembolic events have been associated with rofecoxib compared to naproxen [49]. In another trial rofecoxib was found to be associated with cardiac arrhythmias and renal conditions [50]. However similar adverse effects were not encountered in patients treated with other COX-2 inhibitors [50]. More importantly, several semiselective NSAIDs like diclofenac and meloxicam and nonselective NSAIDs including naproxen and ibuprofen have also been shown to increase the incidences of CVD $[46,51]$. Of these drugs, diclofenac has been shown to increase the occurrences of myocardial infarction and stroke even at lower doses of $<150 \mathrm{mg} /$ day compared to naproxen at doses of $>1000 \mathrm{mg} /$ day [51]. This effect of diclofenac has been attributed to its greater selectivity for COX-2 inhibition. It has also been reported that ibuprofen is associated with CVD comparable to the effect of COX-2 selective inhibitor, celecoxib [20]. It has been suggested that the difference between the effect exhibited by rofecoxib and other NSAIDs of the same class on cardiovascular incidences is due to the distinct chemical properties and prooxidant activity of rofecoxib [52]. The toxic effect of rofecoxib was reportedly due to its ability to reduce the low density lipoprotein (LDL) antioxidant capacity as a result of increased lipid peroxidation [52]. Merck voluntarily withdrew rofecoxib (Vioxx) in 2004. Pfizer was asked by the US Food and Drug Administration (FDA) to withdraw valdecoxib (Bextra) from the market in 2005 because of a higher than expected number of reports of serious and potentially life-threatening skin reactions and deaths.

Naproxen seems to show less cardiovascular events than other commonly used NSAIDs, possibly because it mimics the activity of acetylsalicylic acid (aspirin) by suppressing 
cyclooxygenase platelet thromboxane B2 [53]. However, the role of naproxen in the development of CVD has been controversial. Several studies have reported the cardioprotective effect of the compound [54-56], and on the other hand increased cardiovascular risk has been associated with the use of this NSAID [57-59]. The only NSAID which has not been associated with increased cardiovascular events is aspirin. Aspirin is the only known NSAID which has antithrombotic activity through the inhibition of platelet aggregation in the artery of the heart thereby exhibiting cardioprotective effects [60]. It does so by acetylating the platelet COX-1 and irreversibly suppressing thromboxane A2 production, which is required for platelet aggregation and thrombus formation [60]. Naproxen on the other hand causes reversible inhibition of cyclooxygenase by binding to the enzyme [61]. It was found that inhibition in thromboxane $\mathrm{A} 2$ synthesis was more pronounced (75\%) compared to prostacyclin inhibition (50\%) after the oral administration of $500 \mathrm{mg}$ naproxen in healthy volunteers [61].

\section{NSAIDs and Reactive Oxygen Species Generation}

NSAIDs have been shown to be associated with increased ROS production (Table 3 ). In the heart the main producer of ROS is the mitochondria [62]. Under normal physiological conditions, mitochondria generate ROS as a consequence of aerobic respiration [63]. During aerobic respiration about 5\% of $\mathrm{O}_{2}$ consumed via aerobic reaction is converted into ROS [63]. The mitochondria-dependent overproduction of ROS has been reported under numerous pathological conditions including myocardial heart failure, inflammatory diseases, cancer, hypertension, and diabetes [64-67]. In myocardial heart failure, cardiomyocytes have been shown to be targeted by excessive ROS generation [64].

ROS levels and the redox state of a cell are considered to be important in the dysfunction of various biological signaling pathways. The formation of ROS via the reduction of molecular oxygen or by the oxidation of water leads to the formation of free radicals such as superoxide anion $\left(\mathrm{O}_{2}{ }^{--}\right)$, hydroxyl radical $\left({ }^{\circ} \mathrm{OH}\right)$, and hydrogen peroxide $\left(\mathrm{H}_{2} \mathrm{O}_{2}\right)$. Oxidative stress arises when the oxidant production (sum of all the ROS) surpasses the antioxidant capacity in the cells. Under normal physiological conditions low amounts of $\mathrm{O}_{2}{ }^{-}$ in the cardiomyocytes are converted to the less toxic $\mathrm{H}_{2} \mathrm{O}_{2}$ by the enzyme superoxide dismutase (SOD). The reaction further proceeds by the formation of water by the action of the enzyme catalase or glutathione peroxidase (GPx) system [68]. However, when a homeostatic imbalance between the cellular antioxidant capacity and ROS levels occurs, elevated ROS levels can damage cellular macromolecules including lipids, proteins, and nucleic acids. High levels of ROS also accelerate cell death due to apoptosis as well as necrosis by the activation of poly(adenosine diphosphate ribose) polymerase [69] and thus significantly contribute to the development of various pathological conditions [70].

The generation of ATP in the mitochondria utilizes the electrons from reduced substrates that are transferred to an acceptor molecule of the electron transport chain (ETC).
Leakage of electrons from the mitochondria ETC results in $\mathrm{O}_{2}{ }^{--}$formation [71]. Electron leakage is potentially possible at 9 sites in the ETC; however most of the ROS seem to be associated with complexes I and III which have been well studied [72]. The generation of ROS increases in intact mitochondria as well as in submitochondrial particles due to the oxidation of complex I substrates as a result of inhibiting complex III by antimycin A [72]. On the other hand, rotenone, an inhibitor of complex I, prevented the antimycin A induced ROS generation in mitochondria but not in the submitochondrial particles [72].

The ROS status in the cellular system regulates many biological processes. While increased levels of ROS have been shown to be involved in various pathological conditions, under basal conditions, the generation of free radicals in the heart is needed for cellular responses including regulating myocyte growth and maintaining vascular smooth muscle tone [73]. Basal levels of ROS play an important role in the increase of cell cycle progression and intracellular signaling associated with phosphorylation of several signaling proteins like mitogen-activated protein kinases (MAPKs) and protein kinase B [74]. Under normal physiological conditions, ROS upregulates the Akt signaling pathway and promotes cell survival [75]. ROS also behaves as second messengers in signaling pathways wherein it has been demonstrated that, in the presence of basal ROS levels, tyrosine phosphatase activity is higher relative to its kinases [76]. Ligand stimulation (as in the case of neutrophils, through the binding of cytosolic proteins to a membrane bound oxidase) leads to increased ROS levels and deactivation of tyrosine phosphatases with a consequent increase in the kinase activity [76]. This condition is transient and is reversed by reductions in ROS levels. ROS has also been demonstrated to be involved in the modulation of transcription factors like NF- $\kappa$ B, Hif- 1 [77], and several cardiac transcription factors like SRF, Sp1, AP-1, GATA-4, and MEF2C [78].

Current experimental data suggest that the main mechanism through which NSAIDs exert their anticancer activity is through the generation of ROS leading to oxidative stress and finally apoptosis in cancer cells [79-82]. ROS is accompanied by the activation and inhibition of several signaling pathways associated with cell death and cell survival although controversies exist regarding the role of ROS in the downregulation and upregulation of these pathways [83,84]. The Akt pathway is one of the most important pathways for promoting cell survival and growth, and it has been shown that high ROS levels are lethal and can inactivate the Akt signaling pathway [85]. NF- $\kappa \mathrm{B}$ is an important transcription factor involved in cell survival, inflammation, and stress responses. Its downregulation was demonstrated by exposure of HLEC (human lymphatic endothelial cells) cells to sustained oxidative stress [86]. Increased levels of $\mathrm{H}_{2} \mathrm{O}_{2}$ caused by addition of glucose oxidase to HLECs prevented NF- $\kappa \mathrm{B}(\mathrm{p} 65)$ regulated gene expression by blocking translocation of NF- $\kappa \mathrm{B}$ to the nucleus [86]. NF- $\kappa \mathrm{B}$ regulated gene expression is important for a broad range of physiological processes [86].

Sulindac, a nonselective NSAID, as well as its metabolites, generates ROS in different cancer cell lines [80-82]. Similarly, diclofenac-induced apoptosis of various kinds of cancer cells 


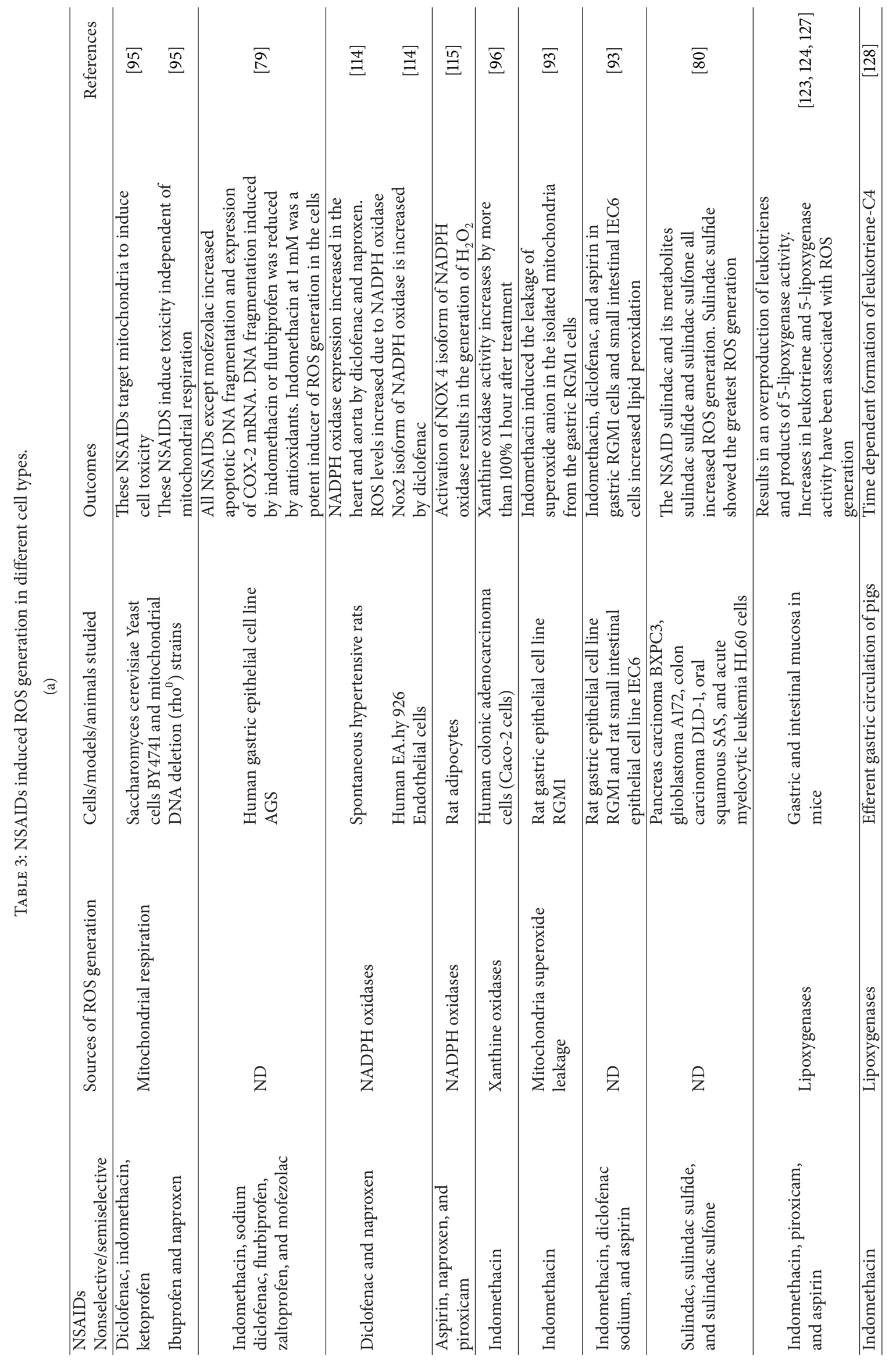




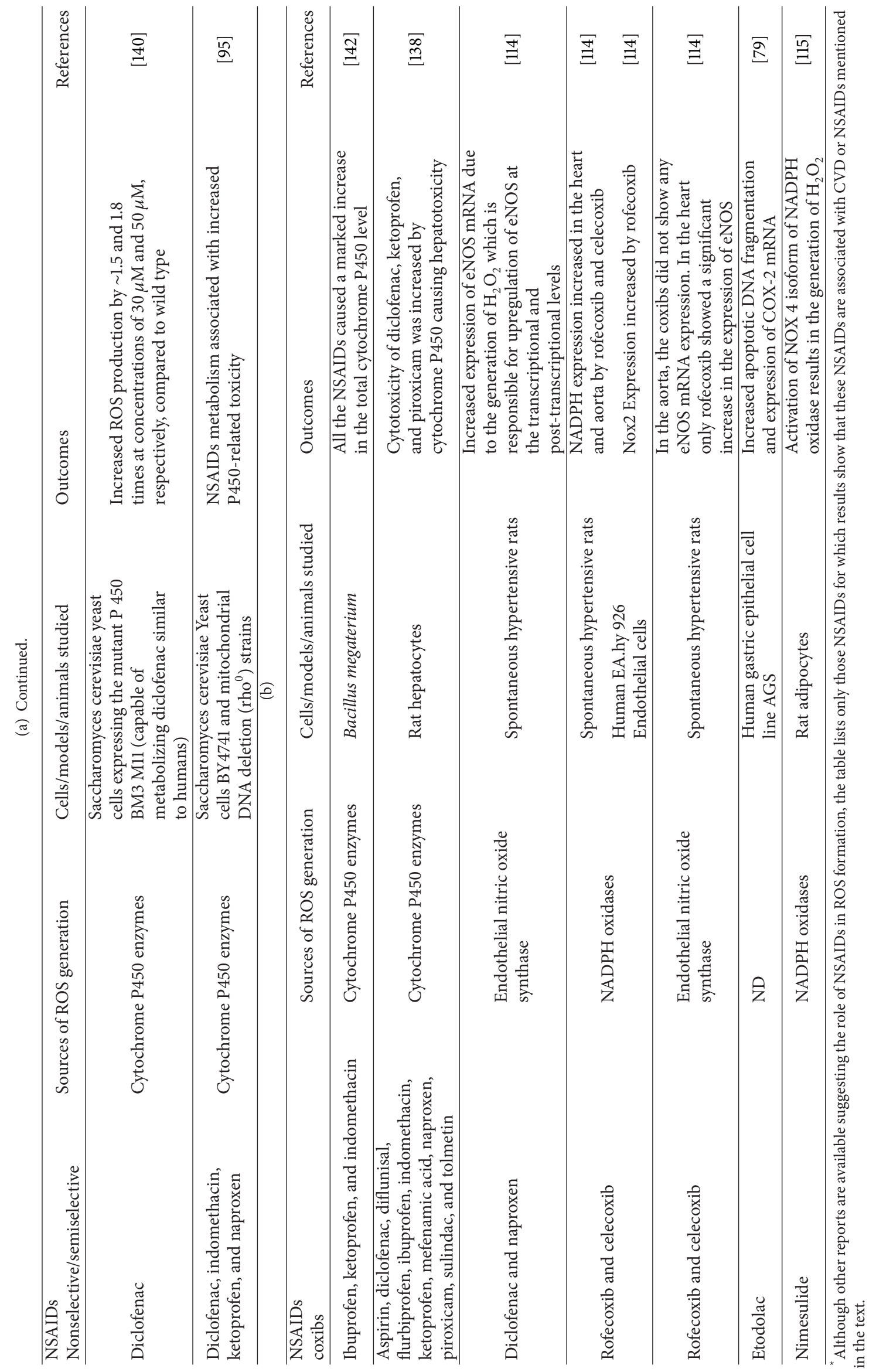


has been reported [87]. This apoptosis is mainly mediated by an increase in the intracellular levels of the ROS [87]. In the cardiovascular system the major sources of ROS generation include the mitochondria, NADPH oxidases, xanthine oxidoreductases, lipoxygenase, cyclooxygenases, nitric oxide synthases, and cytochrome P450 based enzymes (Figure 2). Continuous exposure of cardiovascular cells to oxidative stress associated with elevated ROS levels would result in altered cellular homeostasis which could be an important contributing factor for various cardiovascular conditions. Endothelial cells play a critical role in maintaining vascular homeostasis which is important for the control of many cardiovascular diseases including atherosclerosis and thrombosis [88]. In human umbilical vein endothelial cells (HUVEC), $160 \mu \mathrm{M}$ sulindac induced endothelial apoptosis as determined by a time dependent increase in annexin $\mathrm{V}$ positive cells. Both sulindac and indomethacin significantly increased cleaved poly(ADP-ribose) polymerase (PARP) levels as well as increasing the level of the apoptotic activating factor caspase-3 [89]. The apoptosis in HUVEC cells induced by the NSAIDs was associated with reduced PPAR $\delta$ and 143-3- $\varepsilon$ expression. Under normal conditions $14-3-3-\varepsilon$ binds to phosphorylated Bad inhibiting translocation of Bad to the mitochondria and preventing apoptosis through the mitochondrial pathway. However, sulindac through the suppression of 14-3-3- $\varepsilon$ expression increased Bad translocation to mitochondria thereby inducing apoptosis [89].

\section{Mitochondria Are the Main Target Organelles of the NSAIDs}

It has been shown that the heart is more susceptible to ROS generation induced by drugs like doxorubicin compared to other tissues of the body although the drugs are evenly distributed throughout the body [90]. This is possibly due to high levels of mitochondria in the heart which are the major producers of ROS in the cardiovascular system. Over $90 \%$ of ATP required for the normal functioning of the heart is provided by the mitochondria, which utilizes an efficient oxidative phosphorylation system. ATP production may increase depending upon the requirements of the body, especially at times of excessive physical exertion or other hormonal stimulations [91]. Mitochondria are not only the major producers of the free radicals [72], but excessive generation of ROS in turn targets the mitochondria itself [92]. NSAIDs have been shown to have adverse effects on the mitochondria resulting in the increased production of ROS $[93,94]$. In yeast cells different NSAIDs generated ROS which was associated with delayed growth in wild-type cells [95]. Yeast cells lacking mitochondrial DNA were resistant to a delay in cell growth [95]. More specifically the yeast deletion strains lacking the genes encoding subunits of the mitochondrial complexes III and IV were significantly resistant to diclofenac as well as indomethacin and ketoprofen [95]. This data suggests that mitochondria are the main target organelles through which these compounds exert their toxic effect on these cells. The data also suggest that mitochondrial complex III and/or complex IV are affected by NSAIDs resulting in increased ROS production.

The nonselective NSAID indomethacin has been shown to target mitochondria by directly inhibiting mitochondrial complex 1 of human colonic adenocarcinoma cells. The inhibition of complex I was accompanied by a decrease in the intracellular ATP levels within 10-30 minutes [96]. Studies directly related to mitochondrial dysfunction or damage in the presence of NSAIDs in CVD have not been carried out but are needed (Table 2). Although the mechanism by which NSAIDs can cause mitochondrial dysfunction in cardiomyocytes is not fully understood, preventing increased mitochondrial ROS levels will reduce the adverse effects of elevated ROS levels and may reduce the incidences of CVD caused by NSAIDs.

Apart from the role of NSAIDs in causing cardiovascular incidences, NSAIDs affect other cardiovascular (CV) parameters including left ventricular function, infarct size, and blood pressure.In elderly patients (71.8 \pm 7.6 years) NSAID exposure for $<14$ days showed a significantly higher left ventricular end-systolic dimension $(+1.74 \mathrm{~mm})$ and left ventricular end-diastolic dimension $(+3.69 \mathrm{~mm})$ with a significantly lower fractional shortening $(-6.03 \%)$ when compared to nonNSAID users. Patients with NSAID use for $>14$ days were found to have higher left end-diastolic dimension $(+1.96 \mathrm{~mm})$ but no significant changes in other echocardiographic parameters compared to non-NSAID users [97].

The impact of NSAIDs on infarct size during myocardial infarction has only been investigated by a few laboratories. Indomethacin $(10 \mathrm{mg} / \mathrm{kg})$ was found to increase myocardial infarct size in animals, while ibuprofen $(6.25 \mathrm{mg} / \mathrm{kg} / \mathrm{h}) \mathrm{had}$ the opposite effect [98-100]. The authors suggested that the opposite effect on the infarct size could be attributed to variable doses of the NSAIDs, different degrees of inhibition of prostaglandin and its by-products, and others factors like myocardial oxygen consumption [99]. The same group reported that the frequency of acute infarct expansion syndrome in patients with symptomatic pericarditis treated with indomethacin was $22 \%$ compared to ibuprofen which was only $8 \%$ [101]. The degree of infarct expansion was also greater in patients treated with indomethacin compared to ibuprofen [101].

Another important $\mathrm{CV}$ parameter is hypertension, which is one of the major contributors to the development of CVD. A series of events occur in patients with prolonged high blood pressure including left ventricular hypertrophy, systolic and diastolic dysfunction leading to arrhythmias, and heart failure [102]. Except for aspirin, all NSAIDs could potentially increase blood pressure when taken at doses necessary to alleviate pain and inflammation in both hypertensive and normotensive individuals [39].

NSAIDs through their ability to block COX enzymes lead to the inhibition of renal prostaglandin [103]. Renal prostaglandins modulate several renal functions which include maintaining renal homeostasis and exerting diuretic and natriuretic effects $[104,105]$. Inhibition of the natriuretic effect of COX-2 leads to an increase in sodium retention thereby leading to excess water retention in humans [106]. The inhibition of renal vasodilating prostaglandins induces 
the production of vasoconstricting factors like vasopressin and endothelin-1. This also results in water retention, thereby leading to an increase in the total blood volume and causing altered systolic and diastolic blood pressure [103, 106, 107]. NSAIDs like ibuprofen, indomethacin, and naproxen increase the mean arterial pressure by 5 to $6 \mathrm{mmHg}$ in patients with established hypertension $[108,109]$, which may be enough to raise medical concerns under certain conditions [110]. Additionally, the efficiency of all antihypertensive medications except for calcium channel blockers may be substantially reduced by NSAIDs [40]. Since CV outcomes would have different pathogenesis, increased ROS levels may not be the underlying mechanism for some occurrences of CVD. Other CV related parameters (such as hypertension), which are important for the normal functioning of the heart, are also likely to affect the CV outcome. Although direct comparisons between the different clinical studies shown in Table 2 are not possible, these studies suggest that CVD in NSAID users are influenced by the study populations used (such as with versus without preexisting acute myocardial infarction). Chronic use of NSAIDs by hypertensive individuals has been shown to increase the incidences of myocardial infarction, stroke, and cardiovascular mortality compared to nonchronic NSAID users [111] (Table 2).

\section{NSAIDs and Nicotinamide Adenine Dinucleotide Phosphate-Oxidase}

Apart from mitochondria being the major source of ROS generation, the plasma membrane bound nicotinamide adenine dinucleotide phosphate-oxidase (NADPH oxidase) has also been indicated in the production of ROS in phagocytes like neutrophils and macrophages where it produces an "oxidative burst" of $\mathrm{O}_{2}{ }^{--}$[112]. NADPH oxidases are considered to be the major source of nonmitochondrial ROS generation in many cells. The formation of free radicals is due to the electron transferred from the NADPH to molecular oxygen during the process of phagocytosis. The phagocytic NADPH oxidases play an important role in the host defense mechanism and are involved in killing pathogens ingested during phagocytosis. Apart from the phagocytic NADPH oxidase, in the last decade NADPH oxidase-dependent ROS generation was also identified in nonphagocytic cells including the endothelial cells, vascular smooth muscle cells, and cardiomyocytes of the cardiovascular system [113]. Compared to the phagocytic NADPH oxidases, the nonphagocytic enzymes produce lower amounts of ROS continuously and these levels may increase in the presence of specific extrinsic stimuli [113]. More interestingly, the ROS generated by NADPH oxidases lead to an enhancement in levels of ROS produced from other sources [112]. In one such study, mitochondria and NADPH oxidase 1 isoenzyme (Nox 1) were found to be closely coordinated for sustained generation of ROS leading to oxidative stress and cell death [112]. When human embryonic kidney 293T cells were exposed to serum-free media elevated ROS levels occur within 5 minutes of exposure and persisted for $8 \mathrm{hrs}$ [112]. Utilizing RNA interference Nox isoenzymes were demonstrated to play a role in the induction of serumwithdrawal ROS generation. Although low levels of Nox 2 and Nox 4 did not affect the generation of serum-withdrawal induced ROS generation, low levels of Nox 1 led to a decrease in the ROS formation in these cells at $8 \mathrm{~h}$ (late phase). Using different mitochondrial complex inhibitors such as rotenone and potassium cyanide $(\mathrm{KCN})$ mitochondria were found to play a role in the early phase (first 30 minutes) of ROS generation in the cells exposed to serum-free media [112]. The authors of the study suggested that mitochondrial generation of ROS occurs first, followed by the action of Nox 1 in the serum deprived cell system [112].

The adverse effect of NSAIDs on the cardiovascular system was evident by a study which reported that when male spontaneously hypertensive rats (SHR) were treated either with coxibs or with nonselective NSAIDs like diclofenac and naproxen, the mRNA expression of NOX enzymes 1, 2, and 4 was markedly increased in the heart and aorta [114]. Furthermore the oxidative stress in the heart and aorta of the NSAID treated animals was also elevated along with an increase in the $\mathrm{O}_{2}{ }^{--}$production. Of all the NSAIDs used, diclofenac was the most potent inducer of NADPH oxidases [114]. The role of NADPH oxidases in the generation of ROS in the animals was ascertained by studying the effect of apocynin (an NADPH oxidase inhibitor) in the reversal of the oxidative stress induced by diclofenac [114]. Activation of Nox 4 by NSAIDs like aspirin, naproxen, nimesulide, and piroxicam has also been reported in rat adipocytes resulting in higher production of $\mathrm{H}_{2} \mathrm{O}_{2}$ [115].

\section{NSAIDs and Xanthine Oxidase}

Xanthine oxidoreductase (XOR) is a conserved molybdoflavoenzyme occurring in milk and some tissues including the liver, heart, and small intestine [116]. It has two interconvertible forms: xanthine dehydrogenase $(\mathrm{XDH})$ and xanthine oxidase (XO). Both enzymes catalyze the conversion of hypoxanthine to xanthine and xanthine to uric acid [116]. Although both enzymes are involved in purine degradation, $\mathrm{XO}$ is involved only in the reduction of oxygen while $\mathrm{XDH}$ is involved in the reduction of both oxygen and to a larger extent $\mathrm{NAD}^{+}$. The enzyme XDH has the ability to bind to NAD ${ }^{+}$and, following the oxidation of hypoxanthine to xanthine, reduces $\mathrm{NAD}^{+}$to $\mathrm{NADH}$. On the other hand, $\mathrm{XO}$, not being able to bind to $\mathrm{NAD}^{+}$, produces the free radical, $\mathrm{O}_{2}{ }^{--}$by the transfer of electrons to molecular oxygen. Apart from $\mathrm{O}_{2}{ }^{--}, \mathrm{H}_{2} \mathrm{O}_{2}$ was also shown to be a major free radical generated by $\mathrm{XO}$ [117]. XDH can utilize molecular oxygen and generate ROS but to a lesser extent than XO. Compared to the other sources of ROS generation like mitochondria and NADPH oxidases, the role of XOR as a producer of ROS has been previously overlooked due to its relatively low activity in the heart of animals and human. Recently the role of XOR in mediating various pathological conditions has been demonstrated in the cardiovascular system due to significant increases in cellular XOR levels [118]. This was evident by the inhibition of XOR by compounds like allopurinol and oxypurinol which lead to a decrease in the oxidative tissue damage. Apart from their property of inhibiting XOR, allopurinol and oxypurinol have also been shown to act as free radical scavengers and inhibit lipid peroxidation as well as inducing heat shock proteins 
during times of oxidative stress [118]. Interestingly, a high level of serum uric acid, the end product of XOR, has been shown to be a marker for impaired oxidative metabolism [119].

Although the prevalence of this enzyme has been reported in human hearts $[116,120]$ and although increased levels of uric acid in myocardial heart failure have been reported in the literature [119], the role of NSAIDs on XO in the development of various cardiovascular conditions has not been investigated. In a study to investigate the adverse effect of aspirin on gastric mucosal lining leading to peptic ulcers and intestinal bleeding, rat gastric mucosal cells were incubated with aspirin and cytotoxicity already induced by hypoxanthine/XO was determined [121]. Aspirin was shown to increase the cytotoxicity induced by XO. This increase in cytotoxicity in the presence of hypoxanthine/XO was incorporated with an increase in uric acid levels which suggests ROS generation in these cells. Aspirin had an additive effect to these changes induced by hypoxanthine/XO [121]. In another study, it was seen that indomethacin augmented the $\mathrm{XO}$ activity in human colonic adenocarcinoma cells by more than $100 \%$ upon 60 minutes of exposure which resulted in a time dependent increase in the rate of lipid peroxidation [96].

\section{NSAIDs and Lipoxygenase}

Arachidonic acid that is formed by the action of diacylglycerol and cytosolic phospholipase A2 ( $\left.\mathrm{CPLA}_{2}\right)$ from the membrane phospholipids is a substrate involved in the formation of either prostaglandins and thromboxanes or leukotrienes by the cyclooxygenase and lipoxygenase pathway respectively. The oxidation of arachidonic acid by these enzymes leads to the formation of ROS as the by-product [122]. It was shown that $\mathrm{CPLA}_{2}$-arachidonic acid linked cascade could lead to an increase in ROS generation via the lipoxygenase pathway in the rat fibroblasts [123]. The same group reported that the generation of ROS triggered by tumor necrosis factor- (TNF-) $\alpha$ was mediated by the activation of $\mathrm{CPLA}_{2}$ and the metabolism of arachidonic acid by 5-lipoxygenase [124]. Lipoxygenase has been reported to be involved in the upregulation of NADPH oxidases and increased ROS generation [125].

While NSAIDs are well known to inhibit the cyclooxygenase pathway, they do not inhibit the formation of leukotrienes by the lipoxygenase pathway. NSAIDs increases arachidonic acid levels $[82,126]$ and arachidonic acid itself can increase ROS generation [82]. Increased levels of leukotrienes and other metabolites of the lipoxygenase pathway induced by NSAIDs lead to gastric mucosal damage [127]. The effect of NSAIDs like indomethacin, piroxicam, and aspirin on the development of gastric lesions could be reversed by 5-lipoxygenase inhibitors and leukotriene antagonists [127]. This is consistent with NSAIDs increasing the levels of leukotrienes and products of 5-lipoxygenase activity. Increased occurrences of leukotriene $\mathrm{C} 4$ production were also observed in the gastric circulation induced by indomethacin [128]. The gastric and intestinal lesions in the presence of indomethacin could be reverted by 5lipoxygenase inhibitor. Increased gastric mucosal leukotriene
B4 production was observed in patients suffering from arthritis due to prolonged NSAID treatment [129]. NSAIDs have also been reported to increase the expression of 15 lipoxygenase-1 protein in colorectal cancer cells resulting in the inhibition of cell growth and apoptosis [130]. Although a growing body of evidence supports the idea that NSAIDs upregulate the lipoxygenase pathway, no direct evidence is available on the precise biochemical mechanism by which NSAIDs induced lipoxygenase pathway activity increase the rate of ROS generation. It is possible that the upregulation of the lipoxygenase pathway by NSAIDs may contribute to the net increase in free radicals leading to apoptosis and oxidative cell damage and ultimately cell death.

\section{NSAIDs and Cytochrome P450}

Cytochrome P450 (P450) is a family of biotransformation enzymes involved in critical metabolic processes [131]. They are a class of more than 50 enzymes that catalyze a diverse set of reactions with a wide range of chemically dissimilar substrates [131]. In humans they are primarily membraneassociated proteins that can be found in the inner membrane of the mitochondria or endoplasmic reticulum of cell [132]. They are predominantly expressed in the liver but are present in other tissues of the body as well.This group of proteins belongs to the heme-thiolate enzyme family, and they are involved in the oxidative metabolism of numerous compounds of endogenous and exogenous origin [133]. The role of P450 in drug metabolism has been considered to be a key factor in overcoming the adverse and toxicological effects of drugs [134]. The P450 system has been shown to play an important role in activation of oxygen and ROS generation $[135,136]$. Normally, the active oxygen species are formed in situ during the P450 cycle when it reacts with a substrate. However, uncoupling of the P450 system results in excessive ROS generation and the P450 system being unable to metabolize a substrate, which leads to oxidative stress and subsequently cellular damage $[133,137]$.

Several studies have indicated the role of P450 in toxicity induced by NSAIDs [138-140]. One such NSAID, diclofenac, has been associated with hepatotoxicity due to poor metabolism by P450 [140]. Expression of a drug metabolizing mutant of P450, BM3 M11, in yeast mimicked the oxidative metabolite profiles of diclofenac metabolized by human P450s [141]. The treatment of the yeast cells expressing the mutant BM3 M11 with $30 \mu \mathrm{M}$ and $50 \mu \mathrm{M}$ diclofenac increased the ROS production in the cells significantly by 1.5 and 4 times, respectively, compared to cells not treated with the compound [140]. In another study by the same group, it was shown that diclofenac as well as indomethacin and ketoprofen showed an increase in ROS generation by 1.5-2 times compared to control [95]. On the other hand, P450 has been shown to be upregulated in bacterial cells by NSAIDs like ibuprofen, ketoprofen, and indomethacin by 11.8-fold, 3.9-fold, and 3.0-fold, respectively, relative to control cells [142]. Although no direct evidence is yet available on the involvement of P450 in the generation of ROS by NSAIDs in CVD, experimental data available in other cells suggest 
that NSAIDs may lead to increased ROS levels via the upregulation of P450 in cardiovascular related cells.

\section{NSAIDs and Nitric Oxide Synthases}

Nitric oxide (NO) is among the few gaseous biological messengers and can be synthesized from $l$-arginine by endothelial nitric oxide synthase (eNOS). NO has emerged as a key signaling molecule for maintaining vasodilation and the dysfunction of enzymes associated with $\mathrm{NO}$ production has been implicated in various pathological conditions including CVD, diabetes, and hypertension. When eNOS, which is responsible for regulating vascular homeostasis, is impaired due to a lack of the cofactor tetrahydrobiopterin (BH4), it results in the generation of $\mathrm{O}_{2}{ }^{\cdot-}$ rather than $\mathrm{NO}$ [143]. This state is referred to as the uncoupled state of eNOS and is mainly attributed to the deficiency or lack of $\mathrm{BH} 4$ leading to the generation of free radicals [144]. The depletion of the BH4 can be the result of severe oxidative stress.

A 1.5-fold decrease in plasma nitrite levels was demonstrated in SHR treated with nonselective COX inhibitors and coxibs [114]. Although earlier reports demonstrated a cardioprotective effect of naproxen, a twofold decrease in plasma nitrite levels was observed after treatment of SHR with naproxen suggesting diminished levels of bioactive NO [114]. Additionally, the authors reported an upregulation in the eNOS mRNA expression levels rather than a decrease by diclofenac and naproxen by $>2$-fold. This increase has been attributed to the generation of $\mathrm{H}_{2} \mathrm{O}_{2}$ which increases eNOS expression at the transcriptional and posttranscriptional levels [145]. Diclofenac treated animals showed an increase in $\mathrm{O}_{2}{ }^{--}$production which could be inhibited by the NOS inhibitor $l$-NAME. NSAIDs have also been shown to impair the NO induced vasodilation in healthy individuals possibly due to increased oxidative stress induced by NSAIDs due to the uncoupling of eNOS [114].

\section{NSAIDs, ROS, and Cardiovascular Diseases}

Li et al. investigated the effect of several NSAIDs on free radical generation, NADPH oxidase expression, eNOS expression, and nitrite levels in the rat aorta and heart using a mouse model of hypertension as well as vasodilation in human subjects [114]. As previously described in the sections NSAIDs and nicotinamide adenine dinucleotide phosphateoxidase and NSAIDs and nitric oxide synthases, this study demonstrated that the heart and aorta of the NSAID treated animals showed increased $\mathrm{O}_{2}{ }^{-}$production and oxidative stress. The oxidative stress was due to ROS formation by many enzymes including NADPH oxidases, with diclofenac being the most potent inducer of NADPH oxidases [114].

Rat embryonic H9c2 cardiac cells exposed to celecoxib, at concentrations of $10 \mu \mathrm{M}$ and $100 \mu \mathrm{M}$, demonstrated a decrease in cell viability by $45 \%$ and $92 \%$, respectively, and this was associated with a decrease in the expression levels of the cell survival protein $\mathrm{Bcl} 2$ [146]. However, $1 \mu \mathrm{M}$ of celecoxib had no significant effect on cell viability and was associated with an upregulation of the $\mathrm{Bcl}_{2}$ transcript level by $\sim 30 \%$. This effect of such a low amount of NSAID in the upregulation of cell survival gene expression is rather comparable to the effect of proteasome inhibitors like MG-132 or epoxomicin which at nanomolar ranges has been reported to actually increase the proteasome activity in the pretreated neocortical neurons [147]. Also, this was the first study that measured COX-2 levels directly in the cardiac cells [146]. Although no significant changes in the COX-2 levels were observed in cells treated with $1 \mu \mathrm{M}$ celecoxib, at $10 \mu \mathrm{M}$ the COX-2 levels decreased [146].

Apart from studying the effect of NSAIDs on cardiomyocytes, the role of NSAIDs on the COX enzyme system in platelets is also of prime importance. Platelets are important for the life-saving blood coagulation process [148] and are closely associated with CVD [149]. The aggregation of platelets by agonists like ADP, collagen, thrombin, or epinephrine leads to the formation of thrombus (microaggregate of platelets in fibrin mass), at the site of atherosclerotic plaque fissure on the coronary artery wall of the heart which results in thrombosis or CVD [150]. As expected because of the importance of platelets in CVD, the generation of ROS in platelets has been well-studied [149]. Measurement of $\mathrm{O}_{2}{ }^{-{ }^{-}}$ in platelets activated by thrombin as well as in inactivated platelets demonstrated that thrombin significantly increased the free radical $\left(\mathrm{O}_{2}{ }^{--}\right)$generation by $\sim 40 \%$ in the activated platelets compared to the inactivated platelets [149]. Although this result suggests the possibility of increased ROS formation in activated platelets, no direct role of NSAIDs in the production of ROS in the platelets has been reported [151]. However, it has been demonstrated that selective COX-2 inhibition led to an increase in platelet activation [152]. The increased platelet activation by COX-2 inhibition facilitated thrombotic vessel occlusion after the disruption of the vessel wall supporting a critical role for COX-2 inhibition in platelet activation and aggregation leading to thrombosis [152]. All these effects were accompanied by a decline in the production of endothelial prostacyclin. Diclofenac $(1 \mathrm{mg} / \mathrm{kg})$, a nonselective NSAID, caused significantly increased platelet vessel wall interaction with an increase in the number of adherent platelets on the endothelium of diclofenac treated animals compared to the control group [153]. Thrombotic vessel wall occlusion was also increased in animals treated with diclofenac once the vessel wall had been injured [153]. The direct contribution of ROS induced by NSAIDs to platelet activation and aggregation is yet to be determined. The role of aspirin in cardioprotection by the inhibition of platelet aggregation due to the inhibition of platelet thromboxane A2 is well established $[154,155]$. Similarly, naproxen has also been suggested to have significant antiplatelet effect comparable to that of aspirin [156].

\section{Possible Alternatives to NSAIDs for Reduced Cardiovascular Events}

With the availability of a larger selection of synthetic drugs, people are more aware of the side effects of taking drugs on a regular basis. Nutraceuticals or phytoceuticals are a class of compounds that are derived from plants with fewer or no side effects compared to synthetic drugs [157] but 
with similar and sometimes even better beneficial biological effects [158]. Hyperforin obtained from the herb Hypericum perforatum has been reported to inhibit COX-1 3-18-fold higher when compared to aspirin [158]. Hyperforin is a natural antioxidant and has been shown to inhibit excessive ROS generation [159] as well as prostaglandin synthesis [160]. Several other studies indicate the usefulness of nutraceuticals in cardiovascular protection specifically by the inhibition of COX. Purified anthocyanins (pigment responsible for the color of fruits) have been shown to have an effect on COX activity [161]. While NSAIDs like naproxen $(10 \mu \mathrm{M})$ and ibuprofen $(10 \mu \mathrm{M})$ showed COX-1 inhibition of $47.3 \%$ and $54.3 \%$, respectively, anthocyanins from raspberries $(10 \mu \mathrm{M})$ and blackberries $(10 \mu \mathrm{M})$ showed an inhibition of $45.8 \%$ and $38.5 \%$, respectively. COX-2 inhibition by naproxen and ibuprofen was $39.8 \%$ and $41.3 \%$, for sweet cherries it ranged from $36 \%$ to $48 \%$, and for berries the inhibition was between $31 \%$ and $46 \%$ [161]. The antioxidant activities of the anthocyanins derived from these fruits were also high suggesting the importance of their role in reducing ROS.

In other studies the biologically active compounds of mycelia of Grifola frondosa and Agrocybe aegerita (edible mushrooms) were isolated and studied for their antioxidant and COX activity $[162,163]$. The authors found that that fatty acids like ergosterol, ergostra-4,6,8(14),22-tetraen-3-one, and 1-oleoyl-2-linoleoyl-3-palmitoylglycerol in the case of Grifola frondosa and palmitic acid, ergosterol, 5,8-epidioxy-ergosta6,22-dien-3beta-ol, mannitol, and trehalose in the case of Agrocybe aegerita as determined by spectroscopic analysis were the most potent in inhibiting both COX-1 and COX$2[162,163]$. The efficiency of fish oil omega-3 fatty acids like eicosapentaenoic acid (EPA) and docosahexaenoic acid (DCHA) [164] in preventing CVD, stroke, and high blood pressure is also well established $[165,166]$. It was suggested that fish oils have natural inhibitors which can inhibit COX [167]. While the EPA of fish oils inhibited COX-1 more than COX-2 [168], DCHA was shown to be an effective inhibitor of arachidonic acid induced prostaglandin biosynthesis [169]. Among the other reports available indicating the role of nutraceuticals in the cyclooxygenase pathway, calcitriol (vitamin D) [170], several types of flavonoids [171, 172], and marine derived steroids from formosan soft coral Clavularia viridis [173] all have been reported to affect prostaglandin synthesis. Interestingly most of the compounds exhibit antioxidant properties and inhibit both COX-1 and COX-2 [161, 172] suggesting a role in cardioprotection.

\section{Conclusion}

The review focuses on ROS generated by NSAIDs and its role in CVD. While the number of clinical experiments investigating the effects of NSAIDs on the cardiovascular events has significantly increased over the last two decades, basic research related to the mechanism by which NSAIDs cause cardiovascular dysfunction is limited. High variability in the clinical trials conducted (different populations, dosages, exposure, and types of NSAIDs) has led to results which are difficult to interpret and compare between studies. However, irrespective of the type of NSAID used, increased occurrence of CVD is common. Clinical trials showed that the risk of CVD is higher for coxibs than nonselective NSAIDs probably through the imbalance between prostacyclin/thromboxane levels.

According to the Joint Meeting of the Arthritis Advisory Committee and Drug Safety and Risk Management Advisory Committee, US FDA (2014), patients with a history of myocardial infarction, heart failure, hypertension, and other CV risk factors are at a greater risk of developing cardiovascular diseases due to NSAIDs usage compared to normal individuals [174]. The committee concluded that the increased risk of fatal cardiovascular thrombotic events, myocardial infarction, and stroke by NSAIDS should be lessened by using the lowest effective dose of NSAIDs for the shortest period of time possible.

Interestingly most of the studies that showed the generation of ROS induced by NSAIDs in various types of cells involved nonselective or semiselective NSAIDs (Table 3). However, irrespective of the type of NSAIDs used, either selective COX-2 inhibitors or the nonselective NSAIDs, NSAIDs produced oxidative stress with the nonselective NSAIDs showing a greater degree of ROS generation [114]. Therefore it may be hypothesized that it is through the generation of ROS as well as the upregulation and downregulation of several cell survival pathways that these NSAIDs exert their thrombogenic effect. Although COX-2 inhibitors have been shown to cause CVD some COX-2 inhibitors such as celebrex are currently still used widely, as the FDA determined the benefits of this drug outweigh the potential risks in properly selected and informed patients. The use of COX-2 inhibitors underscores the need for compounds with NSAID like properties without the side effects.

As such, various aspects of NSAID induced cardiotoxicity still need to be investigated, including the significance of NSAID induced lipoxygenase ROS generation in the cardiovascular system, determining if prevention of ROS production reduces CVD and determining if ROS production is needed for pain relief. Answers to these questions will result in substantial improvement on how CVD risk management will be conducted in the future.

\section{Abbreviations}

CVD: Cardiovascular disease

COX-1: Cyclooxygenase-1

COX-2: Cyclooxygenase-2

NSAIDS: Nonsteroidal anti-inflammatory drugs

ROS: Reactive oxygen species.

\section{Conflict of Interests}

The authors declare that there is no conflict of interests regarding the publication of this paper.

\section{Acknowledgment}

This research was carried out using UC Davis Research funds. 


\section{References}

[1] L. J. Crofford, "Use of NSAIDs in treating patients with arthritis," Arthritis Research \& Therapy, vol. 15, supplement 3, article S2, 2013.

[2] Y. I. Cha and R. N. DuBois, "NSAIDs and cancer prevention: targets downstream of COX-2," Annual Review of Medicine, vol. 58, pp. 239-252, 2007.

[3] J. Cuzick, F. Otto, J. A. Baron et al., "Aspirin and nonsteroidal anti-inflammatory drugs for cancer prevention: an international consensus statement," The Lancet Oncology, vol. 10, no. 5, pp. 501-507, 2009.

[4] J. L. Liggett, X. Zhang, T. E. Eling, and S. J. Baek, "Antitumor activity of non-steroidal anti-inflammatory drugs: cyclooxygenase-independent targets," Cancer Letters, vol. 346, no. 2, pp. 217-224, 2014.

[5] F. L. Lanza, F. K. L. Chan, and E. M. M. Quigley, "Guidelines for prevention of NSAID-related ulcer complications," The American Journal of Gastroenterology, vol. 104, no. 3, pp. 728738, 2009.

[6] \&apos; A. Lanas, P. Carrera-Lasfuentes, Y. Arguedas et al., "Risk of upper and lower gastrointestinal bleeding in patients taking nonsteroidal anti-inflammatory drugs, antiplatelet agents, or anticoagulant," Clinical Gastroenterology and Hepatology, 2014.

[7] F. Lapi, L. Azoulay, H. Yin, S. J. Nessim, and S. Suissa, "Concurrent use of diuretics, angiotensin converting enzyme inhibitors, and angiotensin receptor blockers with non-steroidal antiinflammatory drugs and risk of acute kidney injury: nested case-control study," British Medical Journal, vol. 346, no. 7890, Article ID e8525, 2013.

[8] K. A. Taubert, "Cardiology patient pages: can patients with cardiovascular disease take nonsteroidal antiinflammatory drugs?" Circulation, vol. 117, no. 17, pp. e322-e324, 2008.

[9] M. Soyun, J. E. G. Hwang, Z. Cui, and A. V. Gomes, NonSteroidal Anti-Inflammatory Drugs and Increased Risk of Sudden Cardiac Death, Nova Science Publisher, Hauppauge, NY, USA, 2013.

[10] F. E. Silverstein, G. Faich, J. L. Goldstein et al., "Gastrointestinal toxicity with celecoxib vs nonsteroidal anti-inflammatory drugs for osteoarthritis and rheumatoid arthritis: the CLASS study: a randomized controlled trial," The Journal of the American Medical Association, vol. 284, no. 10, pp. 1247-1255, 2000.

[11] L. A. G. Rodr\&apos; iguez and L. B. Tolosa, "Risk of upper gastrointestinal complications among users of traditional NSAIDs and COXIBs in the general population," Gastroenterology, vol. 132, no. 2, pp. 498-506, 2007.

[12] J. L. Goldstein, F. E. Silverstein, N. M. Agrawal et al., "Reduced risk of upper gastrointestinal ulcer complications with celecoxib, a novel COX-2 inhibitor," The American Journal of Gastroenterology, vol. 95, no. 7, pp. 1681-1690, 2000.

[13] A. Helin-Salmivaara, A. Virtanen, R. Vesalainen et al., "NSAID use and the risk of hospitalization for first myocardial infarction in the general population: a nationwide case-control study from Finland," European Heart Journal, vol. 27, no. 14, pp. 1657-1663, 2006.

[14] M. Amer, V. R. Bead, J. Bathon, R. S. Blumenthal, and D. N. Edwards, "Use of nonsteroidal anti-inflammatory drugs in patients with cardiovascular disease: a cautionary tale," Cardiology in Review, vol. 18, no. 4, pp. 204-212, 2010.

[15] A. Rostom, P. Moayyedi, and R. Hunt, "Canadian consensus guidelines on long-term nonsteroidal anti-inflammatory drug therapy and the need for gastroprotection: benefits versus risks,"
Alimentary Pharmacology \& Therapeutics, vol. 29, no. 5, pp. 481496, 2009.

[16] N. A. Nussmeier, A. A. Whelton, M. T. Brown et al., "Complications of the COX-2 inhibitors parecoxib and valdecoxib after cardiac surgery," The New England Journal of Medicine, vol. 352, no. 11, pp. 1081-1091, 2005.

[17] E. M. Antman, J. S. Bennett, A. Daugherty, C. Furberg, H. Roberts, and K. A. Taubert, "Use of nonsteroidal antiinflammatory drugs: an update for clinicians: a scientific statement from the American Heart Association," Circulation, vol. 115, no. 12, pp. 1634-1642, 2007.

[18] M. E. Farkouh and B. P. Greenberg, "An evidence-based review of the cardiovascular risks of nonsteroidal anti-inflammatory drugs," The American Journal of Cardiology, vol. 103, no. 9, pp. $1227-1237,2009$.

[19] D. Mukherjee, S. E. Nissen, and E. J. Topol, "Risk of cardiovascular events associated with selective COX-2 inhibitors," The Journal of the American Medical Association, vol. 286, no. 8, pp. 954-959, 2001.

[20] N. Bhala, J. Emberson, A. Merhi et al., "Vascular and upper gastrointestinal effects of non-steroidal anti-inflammatory drugs: meta-analyses of individual participant data from randomised trials," The Lancet, vol. 382, no. 9894, pp. 769-779, 2013.

[21] T. G. Brock, R. W. McNish, and M. Peters-Golden, "Arachidonic acid is preferentially metabolized by cyclooxygenase- 2 to prostacyclin and prostaglandin E2," The Journal of Biological Chemistry, vol. 274, no. 17, pp. 11660-11666, 1999.

[22] S. U. Monrad, F. Kojima, M. Kapoor et al., "Genetic deletion of mPGES-1 abolishes PGE2 production in murine dendritic cells and alters the cytokine profile, but does not affect maturation or migration," Prostaglandins, Leukotrienes and Essential Fatty Acids, vol. 84, no. 3-4, pp. 113-121, 2011.

[23] R. N. DuBois, S. B. Abramson, L. Crofford et al., "Cyclooxygenase in biology and disease," The FASEB Journal, vol. 12, no. 12, pp. 1063-1073, 1998.

[24] J. Clàiria, "Cyclooxygenase-2 biology," Current Pharmaceutical Design, vol. 9, no. 27, pp. 2177-2190, 2003.

[25] A. Zarghi and S. Arfaei, "Selective COX-2 inhibitors: a review of their structure-activity relationships," Iranian Journal of Pharmaceutical Research, vol. 10, no. 4, pp. 655-683, 2011.

[26] W. L. Smith, R. M. Garavito, and D. L. DeWitt, "Prostaglandin endoperoxide $\mathrm{H}$ synthases (cyclooxygenases)-1 and -2," The Journal of Biological Chemistry, vol. 271, no. 52, pp. 33157-33160, 1996.

[27] D. Ma\&apos; sli\&apos; nska, R. Wo\&apos; zniak, A. Kaliszek, and I. Modelska, "Expression of cyclooxygenase-2 in astrocytes of human bRAin after global ischemia," Folia Neuropathologica, vol. 37, no. 2, pp. 75-79, 1999.

[28] F. Nantel, E. Meadows, D. Denis, B. Connolly, K. M. Metters, and A. Giaid, "Immunolocalization of cyclooxygenase-2 in the macula densa of human elderly," FEBS Letters, vol. 457, no. 3, pp. 475-477, 1999.

[29] N. Zidar, K. Odar, D. Glavac, M. Jerse, T. Zupanc, and D. Stajer, "Cyclooxygenase in normal human tissues-is COX-1 really a constitutive isoform, and COX-2 an inducible isoform?" Journal of Cellular and Molecular Medicine, vol. 13, no. 9, pp. 3753-3763, 2009.

[30] E. Fosslien, "Cardiovascular complications of non-steroidal anti-inflammatory drugs," Annals of Clinical \& Laboratory Science, vol. 35, no. 4, pp. 347-385, 2005. 
[31] J. Song, Q. Chen, and D. Xing, "Enhanced apoptotic effects by downregulating Mcl-1: evidence for the improvement of photodynamic therapy with Celecoxib," Experimental Cell Research, vol. 319, no. 10, pp. 1491-1504, 2013.

[32] Y. Lin, L. Bai, W. Chen, and S. Xu, “The NF- $\kappa$ B activation pathways, emerging molecular targets for cancer prevention and therapy," Expert Opinion on Therapeutic Targets, vol. 14, no. 1, pp. 45-55, 2010.

[33] A. Bank, J. Yu, and L. Zhang, "NSAIDs downregulate Bcl-XL and dissociate BAX and Bcl-X L to induce apoptosis in colon cancer cells," Nutrition and Cancer, vol. 60, supplement 1, pp. 98-103, 2008.

[34] I.-Y. Kim, S.-Y. Park, Y. Kang, D. Thapa, H. G. Choi, and J.-A. Kim, "Role of nonsteroidal anti-inflammatory drug-activated gene-1 in docetaxel-induced cell death of human colorectal cancer cells with different p53 status," Archives of Pharmacal Research, vol. 34, no. 2, pp. 323-330, 2011.

[35] Y.-J. Zhang, Q. Dai, S.-M. Wu et al., "Susceptibility for NSAIDsinduced apoptosis correlates to $\mathrm{p} 53$ gene status in gastric cancer cells," Cancer Investigation, vol. 26, no. 9, pp. 868-877, 2008.

[36] B. B. Aggarwal and S. Shishodia, "Molecular targets of dietary agents for prevention and therapy of cancer," Biochemical Pharmacology, vol. 71, no. 10, pp. 1397-1421, 2006.

[37] M. Yoshida, I. Shiojima, H. Ikeda, and I. Komuro, "Chronic doxorubicin cardiotoxicity is mediated by oxidative DNA damageATM-p53-apoptosis pathway and attenuated by pitavastatin through the inhibition of Racl activity," Journal of Molecular and Cellular Cardiology, vol. 47, no. 5, pp. 698-705, 2009.

[38] D. G. Deavall, E. A. Martin, J. M. Horner, and R. Roberts, "Druginduced oxidative stress and toxicity," Journal of Toxicology, vol. 2012, Article ID 645460, 13 pages, 2012.

[39] T. D. Warner and J. A. Mitchell, "COX-2 selectivity alone does not define the cardiovascular risks associated with nonsteroidal anti-inflammatory drugs," The Lancet, vol. 371, no. 9608, pp. 270-273, 2008.

[40] W. B. White, "Cardiovascular effects of the cyclooxygenase inhibitors," Hypertension, vol. 49, no. 3, pp. 408-418, 2007.

[41] P. Kohli, P. G. Steg, C. P. Cannon et al., "NSAID use and association with cardiovascular outcomes in outpatients with stable atherothrombotic disease," The American Journal of Medicine, vol. 127, no. 1, pp. 53.e1-60.el, 2014.

[42] M. Mamdani, D. N. Juurlink, D. S. Lee et al., "Cyclooxygenase-2 inhibitors versus non-selective non-steroidal antiinflammatory drugs and congestive heart failure outcomes in elderly patients: a population-based cohort study," The Lancet, vol. 363, no. 9423, pp. 1751-1756, 2004.

[43] P. McGettigan and D. Henry, "Cardiovascular risk with non-steroidal anti-inflammatory drugs: systematic review of population-based controlled observational studies," PLoS Medicine, vol. 8, no. 9, Article ID e1001098, 2011.

[44] ADAPT Research Group, "Cardiovascular and cerebrovascular events in the randomized, controlled Alzheimer's disease antiinflammatory prevention trial (ADAPT)," PLoS Clinical Trials, vol. 1, no. 7, article e33, 2006.

[45] S. D. Solomon, J. J. V. McMurray, M. A. Pfeffer et al., "Cardiovascular risk associated with celecoxib in a clinical trial for colorectal adenoma prevention," The New England Journal of Medicine, vol. 352, no. 11, pp. 1071-1080, 2005.

[46] P. M. Kearney, C. Baigent, J. Godwin, H. Halls, J. R. Emberson, and C. Patrono, "Do selective cyclo-oxygenase-2 inhibitors and traditional non-steroidal anti-inflammatory drugs increase the risk of atherothrombosis? Meta-analysis of randomised trials," The British Medical Journal, vol. 332, no. 7553, pp. 1302-1305, 2006.

[47] C. Bombardier, L. Laine, A. Reicin et al., "Comparison of upper gastrointestinal toxicity of rofecoxib and naproxen in patients with rheumatoid arthritis," The New England Journal of Medicine, vol. 343, no. 21, pp. 1520-1528, 2000.

[48] R. S. Bresalier, R. S. Sandler, H. Quan et al., "Cardiovascular events associated with rofecoxib in a colorectal adenoma chemoprevention trial," The New England Journal of Medicine, vol. 352, no. 11, pp. 1092-1102, 2005.

[49] M. A. Konstam, M. R. Weir, A. Reicin et al., "Cardiovascular thrombotic events in controlled, clinical trials of rofecoxib," Circulation, vol. 104, no. 19, pp. 2280-2288, 2001.

[50] J. Zhang, E. L. Ding, and Y. Song, "Adverse effects of cyclooxygenase 2 inhibitors on renal and arrhythmia events: meta-analysis of randomized trials," The Journal of the American Medical Association, vol. 296, no. 13, pp. 1619-1632, 2006.

[51] W. A. Ray, C. Varas-Lorenzo, C. P. Chung et al., "Cardiovascular risks of nonsteroidal antiinflammatory drugs in patients after hospitalization for serious coronary heart disease," Circulation: Cardiovascular Quality and Outcomes, vol. 2, no. 3, pp. 155-163, 2009.

[52] R. P. Mason, M. F. Walter, H. P. McNulty et al., "Rofecoxib increases susceptibility of human LDL and membrane lipids to oxidative damage: a mechanism of cardiotoxicity," Journal of Cardiovascular Pharmacology, vol. 47, supplement 1, pp. S7-S14, 2006.

[53] M. L. Capone, M. G. Sciulli, S. Tacconelli et al., "Pharmacodynamic interaction of naproxen with low-dose aspirin in healthy subjects," Journal of the American College of Cardiology, vol. 45, no. 8, pp. 1295-1301, 2005.

[54] E. Rahme, L. Pilote, and J. LeLorier, "Association between naproxen use and protection against acute myocardial infarction," Archives of Internal Medicine, vol. 162, no. 10, pp. 1111-1115, 2002.

[55] D. H. Solomon, R. J. Glynn, R. Levin, and J. Avorn, "Nonsteroidal anti-inflammatory drug use and acute myocardial infarction," Archives of Internal Medicine, vol. 162, no. 10, pp. 1099-1104, 2002.

[56] D. J. Watson, T. Rhodes, B. Cai, and H. A. Guess, "Lower risk of thromboembolic cardiovascular events with naproxen among patients with rheumatoid arthritis," Archives of Internal Medicine, vol. 162, no. 10, pp. 1105-1110, 2002.

[57] W. A. Ray, C. M. Stein, K. Hall, J. R. Daugherty, and M. R. Griffin, "Non-steroidal anti-inflammatory drugs and risk of serious coronary heart disease: an observational cohort study," The Lancet, vol. 359, no. 9301, pp. 118-123, 2002.

[58] D. J. Graham, D. Campen, R. Hui et al., "Risk of acute myocardial infarction and sudden cardiac death in patients treated with cyclo-oxygenase 2 selective and non-selective nonsteroidal anti-inflammatory drugs: nested case-control study," The Lancet, vol. 365, no. 9458, pp. 475-481, 2005.

[59] M. Mamdani, P. Rochon, D. N. Juurlink et al., "Effect of selective cyclooxygenase 2 inhibitors and naproxen on short-term risk of acute myocardial infarction in the elderly," Archives of Internal Medicine, vol. 163, no. 4, pp. 481-486, 2003.

[60] A. Undas, K. E. Brummel-Ziedins, and K. G. Mann, "Antithrombotic properties of aspirin and resistance to aspirin: beyond strictly antiplatelet actions," Blood, vol. 109, no. 6, pp. 2285-2292, 2007. 
[61] O. Vesterqvist and K. Green, "Effects of naproxen on the in vivo synthesis of thromboxane and prostacyclin in man," European Journal of Clinical Pharmacology, vol. 37, no. 6, pp. 563-565, 1989.

[62] J. F. Turrens and A. Boveris, "Generation of superoxide anion by the NADH dehydrogenase of bovine heart mitochondria," The Biochemical Journal, vol. 191, no. 2, pp. 421-427, 1980.

[63] J. E. Klaunig and L. M. Kamendulis, "The role of oxidative stress in carcinogenesis," Annual Review of Pharmacology and Toxicology, vol. 44, pp. 239-267, 2004.

[64] T. Ide, H. Tsutsui, S. Kinugawa et al., "Mitochondrial electron transport complex I is a potential source of oxygen free radicals in the failing myocardium," Circulation Research, vol. 85, no. 4, pp. 357-363, 1999.

[65] X. Li, P. Fang, J. Mai, E. T. Choi, H. Wang, and X. F. Yang, "Targeting mitochondrial reactive oxygen species as novel therapy for inflammatory diseases and cancers," Journal of Hematology \& Oncology, vol. 6, article 19, 2013.

[66] S. I. Dikalov and Z. Ungvari, "Role of mitochondrial oxidative stress in hypertension," The American Journal of PhysiologyHeart and Circulatory Physiology, vol. 305, no. 10, pp. H1417H1427, 2013.

[67] M. T. Coughlan, D. R. Thorburn, S. A. Penfold et al., "Rageinduced cytosolic ROS promote mitochondrial superoxide generation in diabetes," Journal of the American Society of Nephrology, vol. 20, no. 4, pp. 742-752, 2009.

[68] K. Raedschelders, D. M. Ansley, and D. D. Y. Chen, "The cellular and molecular origin of reactive oxygen species generation during myocardial ischemia and reperfusion," Pharmacology \& Therapeutics, vol. 133, no. 2, pp. 230-255, 2012.

[69] A. J. M. Watson, J. N. Askew, and R. S. P. Benson, "Poly(adenosine diphosphate ribose) polymerase inhibition prevents necrosis induced by $\mathrm{H}_{2} \mathrm{O}_{2}$ but not apoptosis," Gastroenterology, vol. 109, no. 2, pp. 472-482, 1995.

[70] F. J. Giordano, "Oxygen, oxidative stress, hypoxia, and heart failure," The Journal of Clinical Investigation, vol. 115, no. 3, pp. 500-508, 2005.

[71] M. Jastroch, A. S. Divakaruni, S. Mookerjee, J. R. Treberg, and M. D. Brand, "Mitochondrial proton and electron leaks," Essays in Biochemistry, vol. 47, pp. 53-67, 2010.

[72] Q. Chen, E. J. Vazquez, S. Moghaddas, C. L. Hoppel, and E. J. Lesnefsky, "Production of reactive oxygen species by mitochondria: central role of complex III," The Journal of Biological Chemistry, vol. 278, no. 38, pp. 36027-36031, 2003.

[73] H. Y. Lim, W. Wang, J. Chen, K. Ocorr, and R. Bodmer, "ROS regulate cardiac function via a distinct paracrine mechanism," Cell Reports, vol. 7, no. 1, pp. 35-44, 2014.

[74] J. Boonstra and J. A. Post, "Molecular events associated with reactive oxygen species and cell cycle progression in mammalian cells," Gene, vol. 337, pp. 1-13, 2004.

[75] H. Pelicano, R.-H. Xu, M. Du et al., "Mitochondrial respiration defects in cancer cells cause activation of Akt survival pathway through a redox-mediated mechanism," Journal of Cell Biology, vol. 175, no. 6, pp. 913-923, 2006.

[76] T. Finkel, "Oxygen radicals and signaling," Current Opinion in Cell Biology, vol. 10, no. 2, pp. 248-253, 1998.

[77] G. L. Wang, B.-H. Jiang, E. A. Rue, and G. L. Semenza, "Hypoxia-inducible factor 1 is a basic-helix-loop-helix-PAS heterodimer regulated by cellular $\mathrm{O}_{2}$ tension," Proceedings of the National Academy of Sciences of the United States of America, vol. 92, no. 12 , pp. 5510-5514, 1995.
[78] S. K. Law, C. S.-L. Leung, K. L. Yau et al., "Regulation of multiple transcription factors by reactive oxygen species and effects of pro-inflammatory cytokines released during myocardial infarction on cardiac differentiation of embryonic stem cells," International Journal of Cardiology, vol. 168, no. 4, pp. 34583472, 2013.

[79] H. Kusuhara, H. Komatsu, H. Sumichika, and K. Sugahara, "Reactive oxygen species are involved in the apoptosis induced by nonsteroidal anti-inflammatory drugs in cultured gastric cells," European Journal of Pharmacology, vol. 383, no. 3, pp. 331$337,1999$.

[80] M. Adachi, H. Sakamoto, R. Kawamura, W. Wang, K. Imai, and Y. Shinomura, "Nonsteroidal anti-inflammatory drugs and oxidative stress in cancer cells," Histology and Histopathology, vol. 22, no. 4-6, pp. 437-442, 2007.

[81] T. Minami, M. Adachi, R. Kawamura, Y. Zhang, Y. Shinomura, and K. Imai, "Sulindac enhances the proteasome inhibitor bortezomib-mediated oxidative stress and anticancer activity," Clinical Cancer Research, vol. 11, no. 14, pp. 5248-5256, 2005.

[82] C. Giardina and M. S. Inan, "Nonsteroidal anti-inflammatory drugs, short-chain fatty acids, and reactive oxygen metabolism in human colorectal cancer cells," Biochimica et Biophysica Acta-Molecular Cell Research, vol. 1401, no. 3, pp. 277-288, 1998.

[83] E. Barbieri and P. Sestili, "Reactive oxygen species in skeletal muscle signaling," Journal of Signal Transduction, vol. 2012, Article ID 982794, 17 pages, 2012.

[84] I. Dolado and A. R. Nebreda, "AKT and oxidative stress team up to kill cancer cells," Cancer Cell, vol. 14, no. 6, pp. 427-429, 2008.

[85] S.-Y. Kim, S. Bae, K.-H. Choi, and S. An, "Hydrogen peroxide controls Akt activity via ubiquitination/degradation pathways," Oncology Reports, vol. 26, no. 6, pp. 1561-1566, 2011.

[86] M. Wu, Q. Bian, Y. Liu et al., "Sustained oxidative stress inhibits NF- $\kappa$ B activation partially via inactivating the proteasome," Free Radical Biology and Medicine, vol. 46, no. 1, pp. 62-69, 2009.

[87] A. Inoue, S. Muranaka, H. Fujita, T. Kanno, H. Tamai, and K. Utsumi, "Molecular mechanism of diclofenac-induced apoptosis of promyelocytic leukemia: dependency on reactive oxygen species, Akt, Bid, cytochrome c, and caspase pathway," Free Radical Biology \& Medicine, vol. 37, no. 8, pp. 1290-1299, 2004.

[88] C. Michiels, "Endothelial cell functions," Journal of Cellular Physiology, vol. 196, no. 3, pp. 430-443, 2003.

[89] J.-Y. Liou, C.-C. Wu, B.-R. Chen, L. B. Yen, and K. K. Wu, "Nonsteroidal anti-inflammatory drugs induced endothelial apoptosis by perturbing peroxisome proliferator-activated receptor-delta transcriptional pathway," Molecular Pharmacology, vol. 74, no. 5, pp. 1399-1406, 2008.

[90] M. Hacker, "Adverse drug reactions," in Pharmacology: Principles and Practice, M. Hacker, W. S. Messer II, and K. A. Bachmann, Eds., pp. 327-352, Elsevier, New York, NY, USA, 2009.

[91] D. A. Harris and A. M. Das, "Control of mitochondrial ATP synthesis in the heart," The Biochemical Journal, vol. 280, part 3, pp. 561-573, 1991.

[92] S. W. Ballinger, "Mitochondrial dysfunction in cardiovascular disease," Free Radical Biology \& Medicine, vol. 38, no. 10, pp. 1278-1295, 2005.

[93] Y. Nagano, H. Matsui, O. Shimokawa et al., "Rebamipide attenuates nonsteroidal anti-inflammatory drugs (NSAID) induced lipid peroxidation by the manganese superoxide dismutase 
(MnSOD) overexpression in gastrointestinal epithelial cells," Journal of Physiology and Pharmacology, vol. 63, no. 2, pp. 137142, 2012.

[94] R. Moreno-S\&apos; anchez, C. Bravo, C. V\&apos; asquez, G. Ayala, L. H. Silveira, and M. Mart\&apos; inez-Lav\&apos; in, "Inhibition and uncoupling of oxidative phosphorylation by nonsteroidal anti-inflammatory drugs. Study in mitochondria, submitochondrial particles, cells, and whole heart," Biochemical Pharmacology, vol. 57, no. 7, pp. 743-752, 1999.

[95] J. S. van Leeuwen, B. Ünlü, N. P. E. Vermeulen, and J. C. Vos, "Differential involvement of mitochondrial dysfunction, cytochrome P450 activity, and active transport in the toxicity of structurally related NSAIDs," Toxicology in Vitro, vol. 26, no. 2, pp. 197-205, 2012.

[96] C. Carrasco-Pozo, M. Gotteland, and H. Speisky, "Apple peel polyphenol extract protects against indomethacin-induced damage in Caco- 2 cells by preventing mitochondrial complex I inhibition," Journal of Agricultural and Food Chemistry, vol. 59, no. 21, pp. 11501-11508, 2011.

[97] K. E. van den Hondel, M. Eijgelsheim, R. Ruiter, J. C. M. Witteman, A. Hofman, and B. H. C. Stricker, "Effect of shortterm NSAID use on echocardiographic parameters in elderly people: a population-based cohort study," Heart, vol. 97, no. 7, pp. 540-543, 2011.

[98] B. I. Jugdutt, G. M. Hutchins, B. H. Bulkley, B. Pitt, and L. C. Becker, "Effect of indomethacin on collateral blood flow and infarct size in the conscious dog," Circulation, vol. 59, no. 4, pp. 734-743, 1979.

[99] B. I. Jugdutt and L. C. Becker, "Prostaglandin inhibition and myocardial infarct size," Clinical Cardiology, vol. 4, no. 3, pp. 117-124, 1981.

[100] B. I. Jugdutt, G. M. Hutchins, B. H. Bulkley, and L. C. Becker, "Salvage of ischemic myocardium by ibuprofen during infarction in the conscious dog," The American Journal of Cardiology, vol. 46, no. 1, pp. 74-82, 1980.

[101] B. I. Jugdutt and C. A. Basualdo, "Myocardial infarct expansion during indomethacin or ibuprofen therapy for symptomatic post infarction pericarditis. Influence of other pharmacologic agents during early remodelling," The Canadian Journal of Cardiology, vol. 5, no. 4, pp. 211-221, 1989.

[102] M. H. Drazner, "The progression of hypertensive heart disease," Circulation, vol. 123, no. 3, pp. 327-334, 2011.

[103] A. Whelton, "Renal and related cardiovascular effects of conventional and COX-2-specific NSAIDs and non-NSAID analgesics," American Journal of Therapeutics, vol. 7, no. 2, pp. 63-74, 2000.

[104] T. J. Schnitzer, "Cyclooxygenase-2-specific inhibitors: are they safe?” The American Journal of Medicine, vol. 110, no. 1, pp. 46S49S, 2001.

[105] A. N. DeMaria and M. R. Weir, "Coxibs-beyond the GI tract: renal and cardiovascular issues," Journal of Pain and Symptom Management, vol. 25, no. 2, pp. S41-S49, 2003.

[106] C. Stöllberger and J. Finsterer, "Side effects of conventional nonsteroidal anti-inflammatory drugs and celecoxib: more similarities than differences," Southern Medical Journal, vol. 97, no. 2, p. 209, 2004.

[107] W. M. Bennett, W. L. Henrich, and J. S. Stoff, "The renal effects of nonsteroidal anti-inflammatory drugs: summary and recommendations," The American Journal of Kidney Diseases, vol. 28, no. 1, pp. S56-S62, 1996.

[108] J. E. Pope, J. J. Anderson, and D. T. Felson, "A meta-analysis of the effects of nonsteroidal anti-inflammatory drugs on blood pressure," Archives of Internal Medicine, vol. 153, no. 4, pp. 477484, 1993.

[109] A. G. Johnson, T. V. Nguyen, and R. O. Day, "Do nonsteroidal anti-inflammatory drugs affect blood pressure? A meta-analysis," Annals of Internal Medicine, vol. 121, no. 4, pp. 289-300, 1994.

[110] S. A. Grover, L. Coupal, and H. Zowall, “Treating osteoarthritis with cyclooxygenase-2-specific inhibitors: what are the benefits of avoiding blood pressure destabilization?" Hypertension, vol. 45, no. 1, pp. 92-97, 2005.

[111] A. A. Bavry, A. Khaliq, Y. Gong, E. M. Handberg, R. M. CooperDehoff, and C. J. Pepine, "Harmful effects of NSAIDs among patients with hypertension and coronary artery disease," The American Journal of Medicine, vol. 124, no. 7, pp. 614-620, 2011.

[112] S. B. Lee, I. H. Bae, Y. S. Bae, and H. D. Um, "Link between mitochondria and NADPH oxidase 1 isozyme for the sustained production of reactive oxygen species and cell death," The Journal of Biological Chemistry, vol. 281, no. 47, pp. 36228-36235, 2006.

[113] A. C. Cave, A. C. Brewer, A. Narayanapanicker et al., "NADPH oxidases in cardiovascular health and disease," Antioxidants \& Redox Signaling, vol. 8, no. 5-6, pp. 691-728, 2006.

[114] H. Li, M. Hortmann, A. Daiber et al., "Cyclooxygenase 2selective and nonselective nonsteroidal anti-inflammatory drugs induce oxidative stress by up-regulating vascular NADPH oxidases," Journal of Pharmacology and Experimental Therapeutics, vol. 326, no. 3, pp. 745-753, 2008.

[115] H. V\&apos; azquez-Meza, M. Z. de Piña, J. P. Pardo, H. RiverosRosas, R. Villalobos-Molina, and E. Piña, "Non-steroidal antiinflammatory drugs activate NADPH oxidase in adipocytes and raise the $\mathrm{H}_{2} \mathrm{O}_{2}$ pool to prevent cAMP-stimulated protein kinase a activation and inhibit lipolysis," BMC Biochemistry, vol. 14, no. 1, article 13, 2013.

[116] C. E. Berry and J. M. Hare, "Xanthine oxidoreductase and cardiovascular disease: molecular mechanisms and pathophysiological implications," The Journal of Physiology, vol. 555, no. 3, pp. 589-606, 2004.

[117] E. E. Kelley, N. K. H. Khoo, N. J. Hundley, U. Z. Malik, B. A. Freeman, and M. M. Tarpey, "Hydrogen peroxide is the major oxidant product of xanthine oxidase," Free Radical Biology \& Medicine, vol. 48, no. 4, pp. 493-498, 2010.

[118] P. Pacher, A. Nivorozhkin, and C. Szab\&apos;o, “Therapeutic effects of xanthine oxidase inhibitors: renaissance half a century after the discovery of allopurinol," Pharmacological Reviews, vol. 58, no. 1, pp. 87-114, 2006.

[119] F. Leyva, S. Anker, J. W. Swan et al., "Serum uric acid as an index of impaired oxidative metabolism in chronic heart failure," European Heart Journal, vol. 18, no. 5, pp. 858-865, 1997.

[120] J. D. Gladden, B. R. Zelickson, J. L. Guichard et al., "Xanthine oxidase inhibition preserves left ventricular systolic but not diastolic function in cardiac volume overload," The American Journal of Physiology-Heart and Circulatory Physiology, vol. 305, no. 10, pp. H1440-H1450, 2013.

[121] Y. Mitobe, H. Hiraishi, T. Sasai, T. Shimada, and A. Terano, "The effects of aspirin on antioxidant defences of cultured rat gastric mucosal cells," Alimentary Pharmacology and Therapeutics, Supplement, vol. 14, supplement 1, pp. 10-17, 2000.

[122] M. R. Yun, H. M. Park, K. W. Seo, S. J. Lee, D. S. Im, and C. D. Kim, "5-Lipoxygenase plays an essential role in 4HNE-enhanced ROS production in murine macrophages via activation of NADPH oxidase," Free Radical Research, vol. 44, no. 7, pp. 742-750, 2010. 
[123] C.-H. Woo, Z.-W. Lee, B.-C. Kim, K.-S. Ha, and J.-H. Kim, "Involvement of cytosolic phospholipase A2, and the subsequent release of arachidonic acid, in signalling by Rac for the generation of intracellular reactive oxygen species in Rat-2 fibroblasts," Biochemical Journal, vol. 348, no. 3, pp. 525-530, 2000.

[124] C. H. Woo, Y. W. Eom, M. H. Yoo et al., "Tumor necrosis factor-alpha generates reactive oxygen species via a cytosolic phospholipase $\mathrm{A}_{2}$-linked cascade," The Journal of Biological Chemistry, vol. 275, no. 41, pp. 32357-32362, 2000.

[125] K.-J. Cho, J.-M. Seo, and J.-H. Kim, "Bioactive lipoxygenase metabolites stimulation of NADPH oxidases and reactive oxygen species," Molecules and Cells, vol. 32, no. 1, pp. 1-5, 2011.

[126] S. A. Sagi, S. Weggen, J. Eriksen, T. E. Golde, and E. H. Koo, "The non-cyclooxygenase targets of non-steroidal antiinflammatory drugs, lipoxygenases, peroxisome proliferatoractivated receptor, inhibitor of $\kappa \mathrm{B}$ kinase, and $\mathrm{NF} \kappa \mathrm{B}$, do not reduce amyloid $\beta 42$ production," The Journal of Biological Chemistry, vol. 278, no. 34, pp. 31825-31830, 2003.

[127] K. D. Rainsford, "The effects of 5-lipoxygenase inhibitors and leukotriene antagonists on the development of gastric lesions induced by nonsteroidal antiinflammatory drugs in mice," Agents and Actions, vol. 21, no. 3-4, pp. 316-319, 1987.

[128] K. D. Rainsford, "Leukotrienes in the pathogenesis of NSAIDinduced gastric and intestinal mucosal damage," Agents and Actions, vol. 39, pp. C24-C26, 1993.

[129] N. Hudson, M. Balsitis, S. Everitt, and C. J. Hawkey, "Enhanced gastric mucosal leukotriene B4 synthesis in patients taking nonsteroidal anti-inflammatory drugs," Gut, vol. 34, no. 6, pp. 742747, 1993.

[130] I. Shureiqi, D. Chen, J. J. Lee et al., "15-LOX-1: a novel molecular target of nonsteroidal anti-inflammatory drug-induced apoptosis in colorectal cancer cells," Journal of the National Cancer Institute, vol. 92, no. 14, pp. 1136-1142, 2000.

[131] T. Lynch and A. Price, "The effect of cytochrome P450 metabolism on drug response, interactions, and adverse effects," The American Family Physician, vol. 76, no. 3, pp. 391-396, 2007.

[132] K. Berka, T. Hendrychov\&apos; $a$, P. Anzenbacher, and M. Otyepka, "Membrane position of ibuprofen agrees with suggested access path entrance to cytochrome P450 2C9 active site," Journal of Physical Chemistry A, vol. 115, no. 41, pp. 11248-11255, 2011.

[133] R. C. Zangar, D. R. Davydov, and S. Verma, "Mechanisms that regulate production of reactive oxygen species by cytochrome P450," Toxicology and Applied Pharmacology, vol. 199, no. 3, pp. 316-331, 2004.

[134] C. C. Ogu and J. L. Maxa, "Drug interactions due to cytochrome P450," Proceedings (Baylor University. Medical Center), vol. 13, no. 4, pp. 421-423, 2000.

[135] S. Puntarulo and A. I. Cederbaum, "Production of reactive oxygen species by microsomes enriched in specific human cytochrome P450 enzymes," Free Radical Biology \& Medicine, vol. 24, no. 7-8, pp. 1324-1330, 1998.

[136] C. Ioannides and D. F. V. Lewis, "Cytochromes P450 in the bioactivation of chemicals," Current Topics in Medicinal Chemistry, vol. 4, no. 16, pp. 1767-1788, 2004.

[137] D. F. V. Lewis, "Oxidative stress: the role of cytochromes P450 in oxygen activation," Journal of Chemical Technology and Biotechnology, vol. 77, no. 10, pp. 1095-1100, 2002.

[138] M. Jurima-Romet, K. Crawford, and H. S. Huang, "Comparative cytotoxicity of non-steroidal anti-inflammatory drugs in primary cultures of rat hepatocytes," Toxicology in Vitro, vol. 8, no. 1, pp. 55-66, 1994.

[139] J. A. G. Ag\&apos; undez, E. Garc\&apos; ia-Mart\&apos; in, and C. Mart\&apos; inez, "Genetically based impairment in CYP2C8- and CYP2C9-dependent NSAID metabolism as a risk factor for gastrointestinal bleeding: is a combination of pharmacogenomics and metabolomics required to improve personalized medicine?" Expert Opinion on Drug Metabolism \& Toxicology, vol. 5, no. 6, pp. 607-620, 2009.

[140] J. S. van Leeuwen, G. Vredenburg, S. Dragovic, T. F. J. Tjong, J. C. Vos, and N. P. E. Vermeulen, "Metabolism related toxicity of diclofenac in yeast as model system," Toxicology Letters, vol. 200, no. 3, pp. 162-168, 2011.

[141] G. Di Nardo and G. Gilardi, "Optimization of the bacterial cytochrome P450 BM3 system for the production of human drug metabolites," International Journal of Molecular Sciences, vol. 13, no. 12, pp. 15901-15924, 2012.

[142] N. English, V. Hughes, and C. R. Wolf, "Induction of cytochrome P-450 BM-3 (CYP 102) by non-steroidal antiinflammatory drugs in Bacillus megaterium," Biochemical Journal, vol. 316, part 1, pp. 279-283, 1996.

[143] S. Kawashima and M. Yokoyama, "Dysfunction of endothelial nitric oxide synthase and atherosclerosis," Arteriosclerosis, Thrombosis, and Vascular Biology, vol. 24, no. 6, pp. 998-1005, 2004.

[144] N. J. Alp and K. M. Channon, "Regulation of endothelial nitric oxide synthase by tetrahydrobiopterin in vascular disease," Arteriosclerosis, Thrombosis, and Vascular Biology, vol. 24, no. 3, pp. 413-420, 2004.

[145] G. R. Drummond, H. Cai, M. E. Davis, S. Ramasamy, and D. G. Harrison, "Transcriptional and posttranscriptional regulation of endothelial nitric oxide synthase expression by hydrogen peroxide," Circulation Research, vol. 86, no. 3, pp. 347-354, 2000.

[146] K. K. Sakane, C. J. Monteiro, W. Silva et al., "Cellular andmolecular studies of the effects of a selective COX-2 inhibitor celecoxib in the cardiac cell line H9c2 and their correlation with death mechanisms," Brazilian Journal of Medical and Biological Research, vol. 47, no. 1, pp. 50-59, 2014.

[147] C.-S. Lee, L. Y. Tee, T. Warmke et al., "A proteasomal stress response: pre-treatment with proteasome inhibitors increases proteasome activity and reduces neuronal vulnerability to oxidative injury," Journal of Neurochemistry, vol. 91, no. 4, pp. 996-1006, 2004.

[148] M. I. Furman, S. E. Benoit, M. R. Barnard et al., "Increased platelet reactivity and circulating monocyte-platelet aggregates in patients with stable coronary artery disease," Journal of the American College of Cardiology, vol. 31, no. 2, pp. 352-358, 1998.

[149] B. Wachowicz, B. Olas, H. M. Zbikowska, and A. Buczynski, "Generation of reactive oxygen species in blood platelets," Platelets, vol. 13, no. 3, pp. 175-182, 2002.

[150] V. Fuster, J. Badimon, J. H. Chesebro, and J. T. Fallon, "Plaque rupture, thrombosis, and therapeutic implications," Haemostasis, vol. 26, supplement 4, pp. 269-284, 1996.

[151] E. R. Bates and W. C. Lau, "Controversies in antiplatelet therapy for patients with cardiovascular disease," Circulation, vol. 111, no. 17, pp. e267-e271, 2005.

[152] M. A. Buerkle, S. Lehrer, H.-Y. Sohn, P. Conzen, U. Pohl, and F. Krötz, "Selective inhibition of cyclooxygenase-2 enhances platelet adhesion in hamster arterioles in vivo," Circulation, vol. 110, no. 14, pp. 2053-2059, 2004. 
[153] L. Struthmann, N. Hellwig, J. Pircher et al., "Prothrombotic effects of diclofenac on arteriolar platelet activation and thrombosis in vivo," Journal of Thrombosis and Haemostasis, vol. 7, no. 10, pp. 1727-1735, 2009.

[154] N. L. Capurro, L. C. Lipson, R. O. Bonow, R. E. Goldstein, N. R. Shulman, and S. E. Epstein, "Relative effects of aspirin on platelet aggregation and prostaglandin-mediated coronary vasodilation in the dog," Circulation, vol. 62, no. 6, pp. 1221-1227, 1980.

[155] K. Schrör, "Aspirin and platelets: the antiplatelet action of aspirin and its role in thrombosis treatment and prophylaxis," Seminars in Thrombosis and Hemostasis, vol. 23, no. 4, pp. 349356, 1997.

[156] G. A. FitzGerald and C. Patrono, "The coxibs, selective inhibitors of cyclooxygenase-2," The New England Journal of Medicine, vol. 345, no. 6, pp. 433-442, 2001.

[157] V. Brower, "A nutraceutical a day may keep the doctor away," EMBO Reports, vol. 6, no. 8, pp. 708-711, 2005.

[158] D. Albert, I. Zündorf, T. Dingermann, W. E. Müller, D. Steinhilber, and O. Werz, "Hyperforin is a dual inhibitor of cyclooxygenase-1 and 5-lipoxygenase," Biochemical Pharmacology, vol. 64, no. 12, pp. 1767-1775, 2002.

[159] J. Lee, N. Koo, and D. B. Min, "Reactive oxygen species, aging, and antioxidative nutraceuticals," Comprehensive Reviews in Food Science and Food Safety, vol. 3, no. 1, pp. 21-33, 2004.

[160] B. Seaver and J. R. Smith, "Inhibition of COX isoforms by nutraceuticals," Journal of Herbal Pharmacotherapy, vol. 4, no. 2, pp. 11-18, 2004.

[161] N. P. Seeram, R. A. Momin, M. G. Nair, and L. D. Bourquin, "Cyclooxygenase inhibitory and antioxidant cyanidin glycosides in cherries and berries," Phytomedicine, vol. 8, no. 5, pp. 362-369, 2001.

[162] Y. Zhang, G. L. Mills, and M. G. Nair, "Cyclooxygenase inhibitory and antioxidant compounds from the mycelia of the edible mushroom Grifola frondosa," Journal of Agricultural and Food Chemistry, vol. 50, no. 26, pp. 7581-7585, 2002.

[163] Y. Zhang, G. L. Mills, and M. G. Nair, "Cyclooxygenase inhibitory and antioxidant compounds from the fruiting body of an edible mushroom, Agrocybe aegerita," Phytomedicine, vol. 10, no. 5, pp. 386-390, 2003.

[164] P. C. Norris and E. A. Dennis, "Omega-3 fatty acids cause dramatic changes in TLR4 and purinergic eicosanoid signaling," Proceedings of the National Academy of Sciences of the United States of America, vol. 109, no. 22, pp. 8517-8522, 2012.

[165] P. M. Kris-Etherton, W. S. Harris, and L. J. Appel, "Fish consumption, fish oil, omega-3 fatty acids, and cardiovascular disease," Circulation, vol. 106, no. 21, pp. 2747-2757, 2002.

[166] P. M. Kris-Etherton, W. S. Harris, and L. J. Appel, "Fish consumption, fish oil, omega-3 fatty acids, and cardiovascular disease.," Arteriosclerosis, thrombosis, and vascular biology, vol. 23, no. 2, pp. e20-e30, 2003.

[167] L. G. Cleland, M. J. James, and S. M. Proudman, "Fish oil: what the prescriber needs to know," Arthritis Research \& Therapy, vol. 8, article 202, 2006.

[168] M. Wada, C. J. DeLong, Y. H. Hong et al., "Enzymes and receptors of prostaglandin pathways with arachidonic acidderived versus eicosapentaenoic acid-derived substrates and products," The Journal of Biological Chemistry, vol. 282, no. 31, pp. 22254-22266, 2007.

[169] E. J. Corey, C. Shih, and J. R. Cashman, "Docosahexaenoic acid is a strong inhibitor of prostaglandin but not leukotriene biosynthesis," Proceedings of the National Academy of Sciences of the United States of America, vol. 80, no. 12,part 1, pp. 35813584, 1983.

[170] J. Moreno, A. V. Krishnan, D. M. Peehl, and D. Feldman, "Mechanisms of vitamin D-mediated growth inhibition in prostate cancer cells: inhibition of the prostaglandin pathway," Anticancer Research, vol. 26, no. 4, pp. 2525-2530, 2006.

[171] K. A. O’Leary, S. de Pascual-Teresa, P. W. Needs, Y. P. Bao, N. M. O'Brien, and G. Williamson, "Effect of flavonoids and vitamin E on cyclooxygenase-2 (COX-2) transcription," Mutation Research, vol. 551, pp. 245-254, 2004.

[172] A. Bitto, F. Squadrito, N. Irrera et al., "Flavocoxid, a nutraceutical approach to blunt inflammatory conditions," Mediators of Inflammation, vol. 2014, Article ID 790851, 8 pages, 2014.

[173] C.-H. Chang, Z.-H. Wen, S.-K. Wang, and C.-Y. Duh, "New anti-inflammatory steroids from the Formosan soft coral Clavularia viridis," Steroids, vol. 73, no. 5, pp. 562-567, 2008.

[174] U S Food and Drug Administration, "Thrombotic cardiovascular events associated with NSAID use: regulatory history and results of literature search (RCTs)," in Proceedings of the Joint Meeting of the Arthritis Advisory Committee and Drug Safety and Risk Management Advisory Committee, February 2014.

[175] A. Bernareggi, "The pharmacokinetic profile of nimesulide in healthy volunteers," Drugs, vol. 46, no. 1, supplement, pp. 6472, 1993.

[176] E. Kumpulainen, P. Välitalo, M. Kokki et al., "Plasma and cerebrospinal fluid pharmacokinetics of flurbiprofen in children," British Journal of Clinical Pharmacology, vol. 70, no. 4, pp. 557566, 2010.

[177] D. H. Solomon, J. Avorn, T. Stürmer, R. J. Glynn, H. Mogun, and S. Schneeweiss, "Cardiovascular outcomes in new users of coxibs and nonsteroidal antiinflammatory drugs: high-risk subgroups and time course of risk," Arthritis and Rheumatism, vol. 54, no. 5, pp. 1378-1389, 2006.

[178] G. Renda, S. Tacconelli, M. L. Capone et al., "Celecoxib, ibuprofen, and the antiplatelet effect of aspirin in patients with osteoarthritis and ischemic heart disease," Clinical Pharmacology and Therapeutics, vol. 80, no. 3, pp. 264-274, 2006.

[179] C. P. Cannon, S. P. Curtis, G. A. FitzGerald et al., "Cardiovascular outcomes with etoricoxib and diclofenac in patients with osteoarthritis and rheumatoid arthritis in the Multinational Etoricoxib and Diclofenac Arthritis Long-term (MEDAL) programme: a randomised comparison," The Lancet, vol. 368, no. 9549, pp. 1771-1781, 2006.

[180] H. S. B. Baraf, C. Fuentealba, M. Greenwald et al., "Gastrointestinal side effects of etoricoxib in patients with osteoarthritis: results of the etoricoxib versus diclofenac sodium gastrointestinal tolerability and effectiveness (EDGE) trial," The Journal of Rheumatology, vol. 34, no. 2, pp. 408-420, 2007.

[181] M. E. Farkouh, J. D. Greenberg, R. V. Jeger et al., "Cardiovascular outcomes in high risk patients with osteoarthritis treated with ibuprofen, naproxen or lumiracoxib," Annals of the Rheumatic Diseases, vol. 66, no. 6, pp. 764-770, 2007.

[182] S. S. Jick, J. A. Kaye, and H. Jick, "Diclofenac and acute myocardial infarction in patients with no major risk factors," British Journal of Clinical Pharmacology, vol. 64, no. 5, pp. 662667, 2007.

[183] K. Krueger, L. Lino, R. Dore et al., "Gastrointestinal tolerability of etoricoxib in rheumatoid arthritis patients: results of the etoricoxib vs diclofenac sodium gastrointestinal tolerability and effectiveness trial (EDGE-II)," Annals of the Rheumatic Diseases, vol. 67, no. 3, pp. 315-322, 2008. 
[184] S. D. Solomon, J. Wittes, P. V. Finn et al., "Cardiovascular risk of celecoxib in 6 randomized placebo-controlled trials: the cross trial safety analysis," Circulation, vol. 117, no. 16, pp. 2104-2113, 2008.

[185] T.-P. van Staa, S. Rietbrock, E. Setakis, and H. G. M. Leufkens, "Does the varied use of NSAIDs explain the differences in the risk of myocardial infarction?" Journal of Internal Medicine, vol. 264, no. 5, pp. 481-492, 2008.

[186] B. Combe, G. Swergold, J. McLay et al., "Cardiovascular safety and gastrointestinal tolerability of etoricoxib vs diclofenac in a randomized controlled clinical trial (The MEDAL study)," Rheumatology, vol. 48, no. 4, pp. 425-432, 2009.

[187] C. L. Roumie, N. N. Choma, L. Kaltenbach, E. F. Mitchel Jr., P. G. Arbogast, and M. R. Griffin, "Non-aspirin NSAIDs, cyclooxygenase-2 inhibitors and risk for cardiovascular eventsstroke, acute myocardial infarction, and death from coronary heart disease," Pharmacoepidemiology and Drug Safety, vol. 18, no. 11, pp. 1053-1063, 2009.

[188] E. L. Fosbøl, G. H. Gislason, S. Jacobsen et al., "Risk of myocardial infarction and death associated with the use of nonsteroidal anti-inflammatory drugs (NSAIDs) among healthy individuals: a nationwide cohort study," Clinical Pharmacology and Therapeutics, vol. 85, no. 2, pp. 190-197, 2009.

[189] M. C. Becker, T. H. Wang, L. Wisniewski et al., "Rationale, design, and governance of Prospective Randomized Evaluation of Celecoxib Integrated Safety versus Ibuprofen Or Naproxen (PRECISION), a cardiovascular end point trial of nonsteroidal antiinflammatory agents in patients with arthritis," The American Heart Journal, vol. 157, no. 4, pp. 606-612, 2009.

[190] G. H. Gislason, J. N. Rasmussen, S. Z. Abildstrom et al., "Increased mortality and cardiovascular morbidity associated with use of nonsteroidal anti-inflammatory drugs in chronic heart failure," Archives of Internal Medicine, vol. 169, no. 2, pp. 141-149, 2009.

[191] M. W. van der Linden, S. van der Bij, P. Welsing, E. J. Kuipers, and R. M. C. Herings, "The balance between severe cardiovascular and gastrointestinal events among users of selective and nonselective non-steroidal anti-inflammatory drugs," Annals of the Rheumatic Diseases, vol. 68, no. 5, pp. 668-673, 2009.

[192] M. Schmidt, C. F. Christiansen, F. Mehnert, K. J. Rothman, and H. T. Sørensen, "Non-steroidal anti-inflammatory drug use and risk of atrial fibrillation or flutter: population based case-control study," The British Medical Journal, vol. 343, no. 7814, Article ID d3450, 2011.

[193] A.-M. Schjerning Olsen, E. L. Fosbøl, J. Lindhardsen et al., "Duration of treatment with nonsteroidal anti-inflammatory drugs and impact on risk of death and recurrent myocardial infarction in patients with prior myocardial infarction: a nationwide cohort study," Circulation, vol. 123, no. 20, pp. 2226-2235, 2011.

[194] A. M. S. Olsen, E. L. Fosbøl, J. Lindhardsen et al., "Long-term cardiovascular risk of nonsteroidal anti-inflammatory drug use according to time passed after first-time myocardial infarction: a nationwide cohort study," Circulation, vol. 126, no. 16, pp. 1955-1963, 2012. 


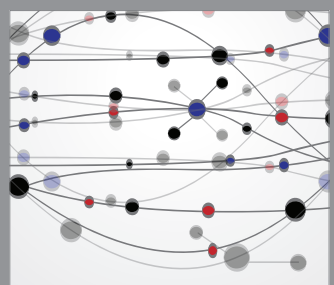

The Scientific World Journal
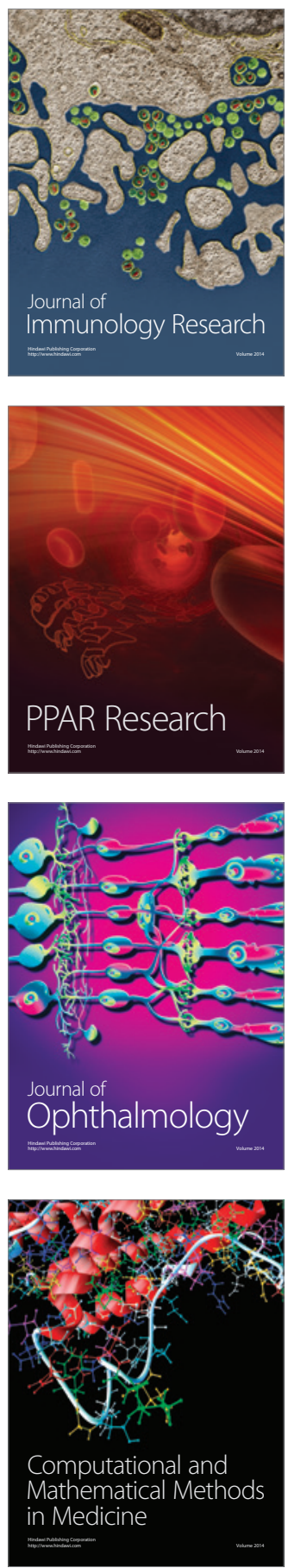

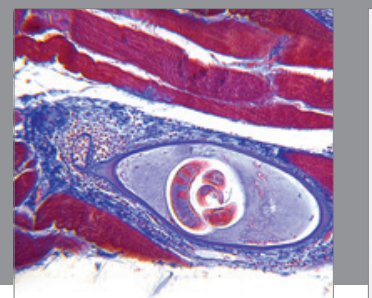

Gastroenterology

Research and Practice
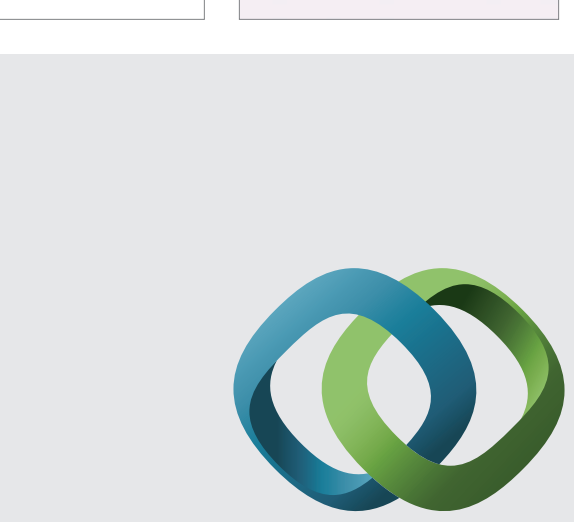

\section{Hindawi}

Submit your manuscripts at

http://www.hindawi.com
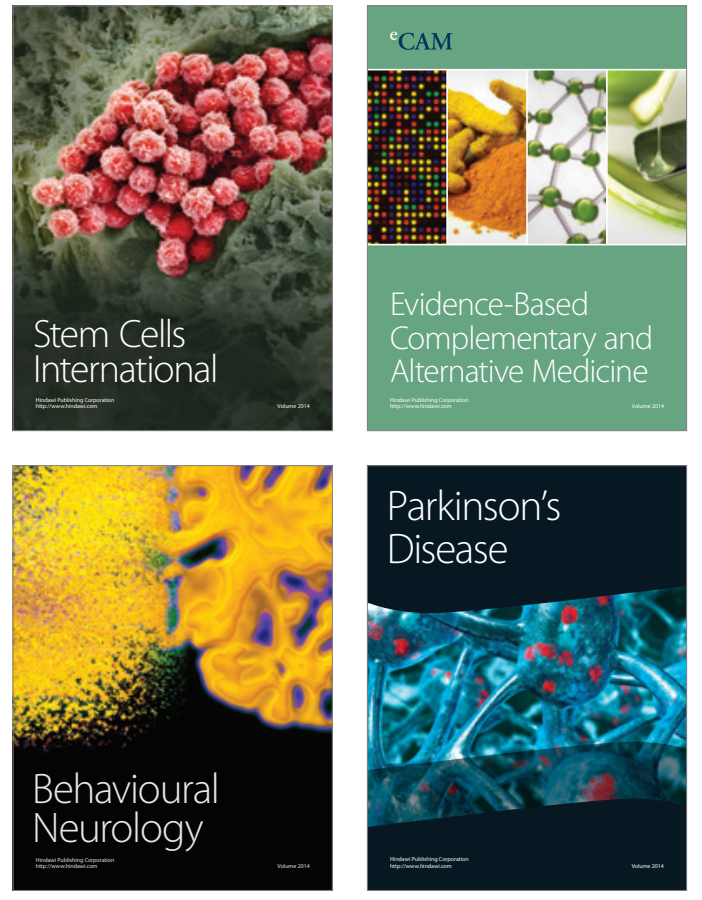
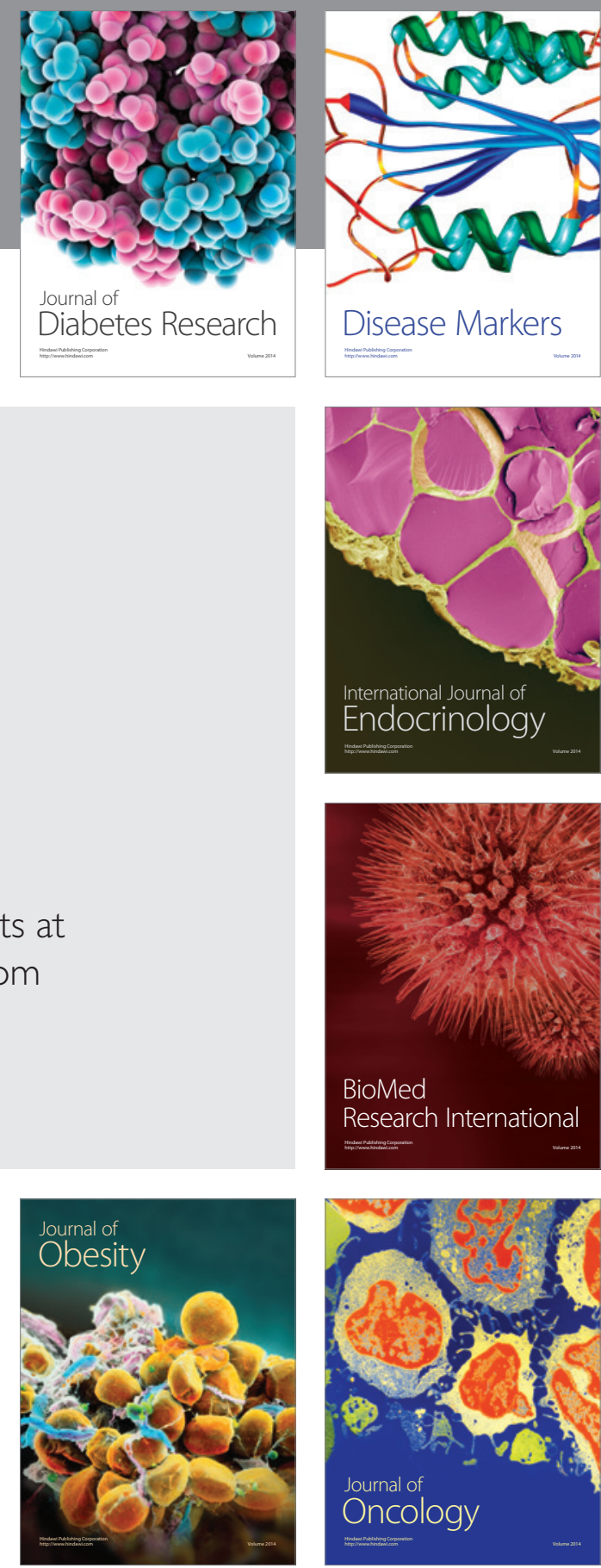

Disease Markers
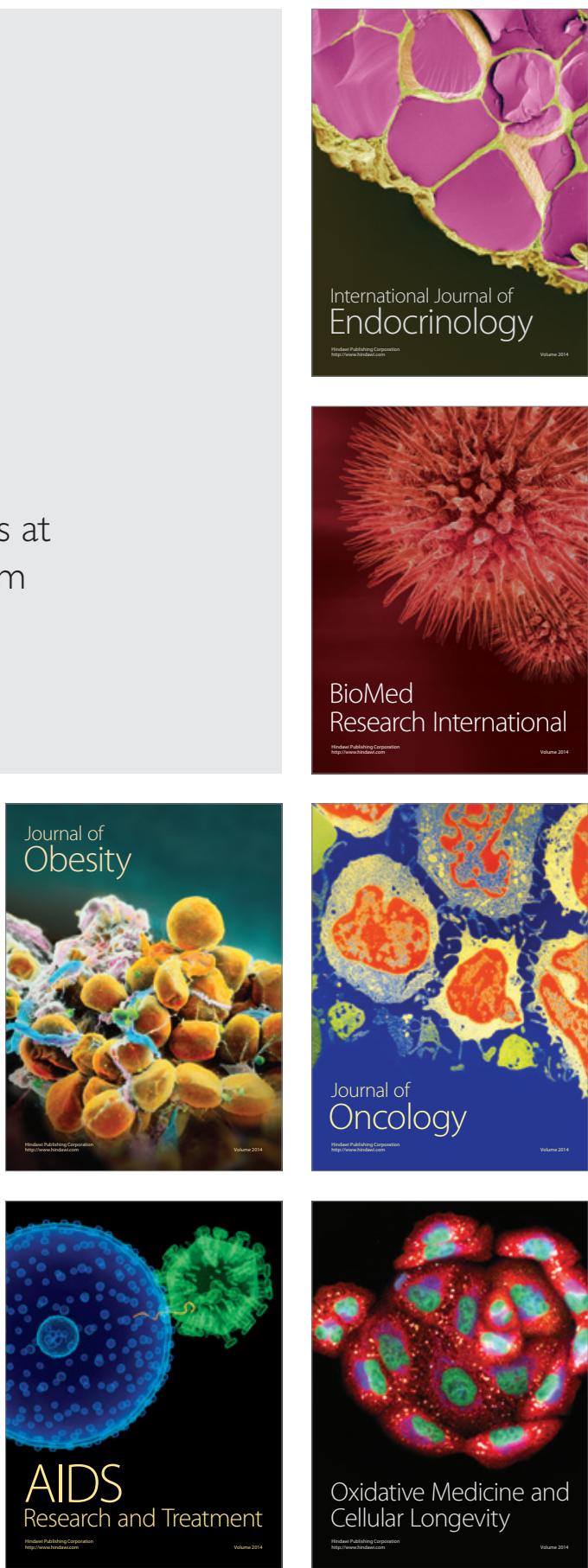